\title{
Unintegrated gluon distributions and Higgs boson production in proton-proton collisions
}

\author{
M. Euszczak ${ }^{2}$ and A. Szczurek ${ }^{1,2}$ \\ ${ }^{1}$ Institute of Nuclear Physics PAN \\ PL-31-342 Cracow, Poland \\ ${ }^{2}$ University of Rzeszów \\ PL-35-959 Rzeszów, Poland
}

\begin{abstract}
Inclusive cross sections for Higgs boson production in proton-proton collisions are calculated in the formalism of unintegrated gluon distributions (UGDF). Different UGDF from the literature are used. Although they were constructed in order to describe the HERA deepinelastic scattering $F_{2}$ data, they lead to surprisingly different results for Higgs boson production. We present both two-dimensional invariant cross section as a function of Higgs boson rapidity and transverse momentum, as well as corresponding projections on rapidity or transverse momentum. We quantify the differences between different UGD's by applying different cuts on interrelations between transverse momentum of Higgs and transverse momenta of both fusing gluons. We focus on large rapidity region. The interplay of the gluon-gluon fusion and weak-boson fusion in rapidity and transverse momentum is discussed. We find that above $p_{T} \sim 50-100 \mathrm{GeV}$ the weak-gauge-boson fusion dominates over gluon-gluon fusion.
\end{abstract}

PACS 12.38.Bx,12.38.Cy,13.85.Qk,14.70.Hp,14.80.Bn

\section{Introduction}

Recently unintegrated gluon (parton) distributions became a useful and intuitive phenomenological language for applications to many high-energy reactions (see e.g. [1, 2] and references therein). Mostly the HERA $F_{2}$ data was used to test or tune different models of UGDF's. However, the structure function data are not the best tool to verify UGDF, in particular its dependence on gluon transverse momentum, because it enters to the $\gamma^{*} p$ total cross 
section in an integrated way. UGDF's have been used recently to describe jet correlations 3], correlations in heavy quark photoproduction 4], total cross section for Higgs production [5], inclusive spectra of pions in proton-proton collisions [6] or even nucleus-nucleus collisions [7]. It is rather obvious that differential cross sections seem a much better tool than total or integrated cross sections to verify UGDF's.

Many unintegrated gluon distributions in the literature are ad hoc parametrizations of different sets of experimental data rather than derived from QCD. An example of a more systematic approach, making use of familiar collinear distributions can be found in Ref. [8]. Recently Kwieciński and collaborators 9, 10, 11] have shown how to solve so-called CCFM equations by introducing unintegrated parton distributions in the space conjugated to the transverse momenta 9]. We present results for inclusive Higgs production based on unintegrated gluon distributions obtained by solving a set of coupled equations [11. Recently these parton distributions were tested for inclusive gauge boson production in proton-antiproton collisions [12] and for charm-anticharm correlations in photoproduction [4].

While in the gauge boson production one tests mainly quark and antiquark (unintegrated) distributions at scales $\mu^{2} \sim M_{W}^{2}, M_{Z}^{2}$, in the charmquark photoproduction one tests mainly gluon distributions at scales $\mu^{2} \sim$ $m_{c}^{2}$. The nonperturbative aspect of UPDF's can be tested for soft pion production in proton-proton collisions [13].

Different ideas based on perturbative and nonperturbative QCD have been used in the literature to obtain the unintegrated gluon distributions in the small-x region. Since almost all of them were constructed to describe the HERA data it is necessary to test these distributions in other high-energy processes in order to verify the underlying concepts and/or approximations applied. It is the aim of this paper to show predictions of these quite different UGDF's for Higgs production at LHC at CERN although we are aware of the fact that a real test against future experimental data may be extremely difficult. We compare and analyze two-dimensional distributions for inclusive Higgs production in rapidity and transverse momentum $\left(y, p_{t}\right)$ to study a potential for such an analysis in the future. We focus not only on midrapidities but also try to understand a potential for studying UGDF's in more forward or backward rapidity regions. The results of the gluon-gluon fusion are compared with other mechanisms of Higgs boson production such as WW fusion for instance. 


\section{Formalism}

There are several mechanisms of the Higgs boson production which have been discussed in the literature. Provided that the Higgs mass is larger than $100 \mathrm{GeV}$ and smaller than $600 \mathrm{GeV}$ the gluon-gluon fusion (see Fig 1) is the dominant mechanism of Higgs boson production at LHC energies [14]. The WW and ZZ fusion is the second important mechanism for the light-Higgs scenario. Often the so-called associated Higgs boson production $(H W, H Z$ or $H t \bar{t}$ ) is considered as a good candidate for the discovery of the Higgs boson. The contribution of the associated production to the inclusive cross section is, however, rather small. In the present paper we concentrate on the inclusive cross section and in particular on its dependence on rapidity and/or transverse momentum of the Higgs boson.

\subsection{Gluon-gluon fusion}

In the leading-order collinear factorization approach the Higgs boson has a zero transverse momentum. In the collinear approach the finite transverse momenta are generated only at next-to-leading order. However, the fixed-order approach is not useful for small transverse momenta and rather a resummation method must be used [16]. Recently Kwieciński, starting from the CCFM equation [15], has proposed a new method of resummation [17] based on unintegrated gluon distributions.

Limiting to small transverse momenta of the Higgs boson, i.e. small transverse momenta of the fusing gluons, the on-shell approximation for the matrix element seems a good approximation. There are also some technical reasons, to be discussed later, to stay with the on-shell approximation. In the on-shell approximation for the $g g \rightarrow H$ transition matrix element ${ }^{1}$ the leading order cross section in the unintegrated gluon distribution formalism reads $^{2}$

$$
\frac{d \sigma^{H}}{d y d^{2} p_{t}}=\sigma_{0}^{g g \rightarrow H} \int f_{g / 1}\left(x_{1}, \kappa_{1}^{2}, \mu^{2}\right) f_{g / 2}\left(x_{2}, \kappa_{2}^{2}, \mu^{2}\right) \delta^{2}\left(\vec{\kappa}_{1}+\vec{\kappa}_{2}-\vec{p}_{t}\right) \frac{d^{2} \kappa_{1}}{\pi} \frac{d^{2} \kappa_{2}}{\pi}
$$

In the equation above the delta function assures conservation of transverse momenta in the gluon-gluon fusion subprocess. The $1 / \pi$ factors are due to the definition of UGDF's. The momentum fractions should be calculated as

\footnotetext{
${ }^{1}$ There is a disagreement in the literature [19, 20] how to include the off-shell effects.

${ }^{2}$ Some of UGDF's from the literature depend only on longitudinal momentum fraction and transverse momentum of the virtual gluon. To keep formulae below general we allow for a scale parameter needed in some distributions.
} 
$x_{1,2}=\frac{m_{t, H}}{\sqrt{s}} \exp ( \pm y)$, where in comparison to the collinear case $M_{H}$ is replaced by the Higgs transverse mass $m_{t, H}$. If we neglect transverse momenta and perform the following formal substitutions

$$
\begin{aligned}
& f_{g / 1}\left(x_{1}, \kappa_{1}^{2}, \mu^{2}\right) \rightarrow x_{1} g_{1}\left(x_{1}, \mu^{2}\right) \delta\left(\kappa_{1}^{2}\right), \\
& f_{g / 2}\left(x_{2}, \kappa_{2}^{2}, \mu^{2}\right) \rightarrow x_{2} g_{2}\left(x_{2}, \mu^{2}\right) \delta\left(\kappa_{2}^{2}\right) .
\end{aligned}
$$

we recover the well known leading-order formula

$$
\frac{d \sigma^{H}}{d y d^{2} p_{t}}=\sigma_{0}^{g g \rightarrow H} x_{1} g_{1}\left(x_{1}, \mu^{2}\right) x_{2} g_{2}\left(x_{2}, \mu^{2}\right) \delta^{2}\left(\vec{p}_{t}\right) .
$$

The off-shell effects could be taken into account by inserting the $\gamma^{*} \gamma^{*} \rightarrow$ $H$ off-shell cross section (corresponding to the matrix element squared $\left|\mathcal{M}^{g g \rightarrow H}\left(\vec{\kappa}_{1}, \vec{\kappa}_{2}\right)\right|^{2}$ ) under the integral in the formula (11) above. This will be discussed in more detail in a separate subsubsection.

There are a few conventions for UGDF in the literature. In the convention used throughout the present paper the unintegrated gluon distributions have dimension of $\mathrm{GeV}^{-2}$ and fulfil the approximate relation

$$
\int_{0}^{\mu^{2}} f_{g}\left(x, \kappa^{2}\left(, \mu^{2}\right)\right) d \kappa^{2} \approx x g_{\text {coll }}\left(x, \mu^{2}\right)
$$

where $g_{\text {coll }}\left(x, \mu^{2}\right)$ is the familiar conventional (integrated) gluon distribution. The scale $\mu^{2}$ in UGDF above is optional. In the effective Lagrangian approximation and assuming infinitely heavy top quark the cross section parameter $\sigma_{0}^{g g \rightarrow H}$ is given by [29]

$$
\sigma_{0}^{g g \rightarrow H}=\frac{\sqrt{2} G_{F}}{576 \pi} \alpha_{s}^{2}\left(\mu_{r}^{2}\right) .
$$

In the following we shall take $\mu_{r}^{2}=M_{H}^{2}$. Above we have assumed implicitly that the fusing gluons are on-mass-shell. In general, the fusing gluons are off-mass-shell. This effect was analyzed in detail in the production of Higgs associated with two jets [30]. The effect found there is small provided $M_{H}<$ $2 m_{t}$.

The UGDF's are the main ingredients in evaluating the inclusive cross section for Higgs production. Depending on the approach, some UGDF's 24, 7, 25, 26] in the literature depend on two variables - longitudinal momentum fraction $x$ and transverse momentum $\kappa^{2}$, some in addition depend on a scale parameter [8, 9, 10, 27]. In the last case the scale $\mu^{2}$ is taken here as $M_{H}^{2}$ or $\xi M_{H}^{2}$, where $\xi$ is some factor. 
The seemingly 4-dimensional integrals in Eq.(1) can be written as 2dimensional integrals after a suitable change of variables $\vec{\kappa}_{1}, \vec{\kappa}_{2} \rightarrow \vec{p}_{t}, \vec{q}_{t}$, where $\vec{p}_{t}=\vec{\kappa}_{1}+\vec{\kappa}_{2}$ and $\vec{q}_{t}=\vec{\kappa}_{1}-\vec{\kappa}_{2}$. Then

$$
\frac{d \sigma^{H}}{d y d^{2} p_{t}}=\frac{\sigma_{0}^{g g \rightarrow H}}{(2 \pi)^{2}} \int f_{g / 1}\left(x_{1}, \kappa_{1}^{2}, \mu^{2}\right) f_{g / 2}\left(x_{2}, \kappa_{2}^{2}, \mu^{2}\right) d^{2} q_{t},
$$

where $\vec{\kappa}_{1}=\vec{p}_{t} / 2+\vec{q}_{t} / 2$ and $\vec{\kappa}_{2}=\vec{p}_{t} / 2-\vec{q}_{t} / 2$. The integrand of this "reduced" 2-dimensional integral in $\overrightarrow{q_{t}}=\overrightarrow{\kappa_{1}}-\overrightarrow{\kappa_{2}}$ is generally a smooth function of $q_{t}$ and corresponding azimuthal angle $\phi_{q_{t}}$.

\subsubsection{Unintegrated gluon distributions}

In the present analysis we shall use different unintegrated gluon distributions from the literature. We include gluon distributions corresponding to a simple saturation model used by Golec-Biernat and Wüsthoff to describe the HERA deep-inelastic data 24] (GBW), a saturation model of Kharzeev and Levin used to describe rapidity distributions of charged particles [7] (KL) ${ }^{3}$, Balitskij-Fadin-Kuraev-Lipatov (BFKL)-type UGDF [25] and three other distributions in the transverse-momentum space [27], 8] (KMR) and [26] as well as the Kwieciński UGDF in the b-space. A more detailed description of almost all distributions mentioned above can be found e.g. in Ref. [6]. The Kwieciński UGDF as the only one defined in the b-space requires a separate discussion. It will be shown below that the formulae for the inclusive cross section for Higgs boson production via gluon-gluon fusion can be written in the equivalent way also in terms of distributions in the b-space.

\subsubsection{Kwieciński gluon distribution and the inclusive cross section}

Taking the following representation of the $\delta$ function

$$
\delta^{2}\left(\overrightarrow{\kappa_{1}}+\overrightarrow{\kappa_{2}}-\overrightarrow{p_{t}}\right)=\frac{1}{(2 \pi)^{2}} \int d^{2} b \exp \left[\left(\overrightarrow{\kappa_{1}}+\overrightarrow{\kappa_{2}}-\overrightarrow{p_{t}}\right) \vec{b}\right]
$$

the formula (6) can be written in the equivalent way in terms of gluon distributions in the space conjugated to the gluon transverse momentum ${ }^{4}$

$$
\frac{d \sigma^{H}}{d y d^{2} p_{t}}=\sigma_{0}^{g g \rightarrow H} \int \tilde{f}_{g / 1}\left(x_{1}, b, \mu^{2}\right) \tilde{f}_{g / 2}\left(x_{2}, b, \mu^{2}\right) J_{0}\left(p_{t} b\right) 2 \pi b d b,
$$

\footnotetext{
${ }^{3}$ The normalization of the gluon distributions was fixed in [6] to reproduce the HERA data.

${ }^{4}$ The simple form of the formula below would not be possible with off-shell effects, i.e. when $\left|\mathcal{M}^{g g \rightarrow H}\right|^{2}$ is a function of $\vec{\kappa}_{1}$ and $\vec{\kappa}_{2}$.
} 
where

$$
\tilde{f}_{g}\left(x, b, \mu^{2}\right)=\int_{0}^{\infty} d \kappa_{t} \kappa_{t} J_{0}\left(\kappa_{t} b\right) f_{g}\left(x, \kappa_{t}^{2}, \mu^{2}\right) .
$$

For most of unintegrated gluon distributions formula (6) is used. Formula (8) is used when applying the Kwieciński unintegrated distributions obtained as a solution of his equations in the b-space. The b-space approach proposed by Kwieciński is very convenient to introduce the nonperturbative effects like intrinsic (nonperturbative) transverse momentum distributions of partons in nucleons. It seems reasonable, at least in the first approximation, to include the nonperturbative effects in the factorizable way

$$
\tilde{f}_{g}\left(x, b, \mu^{2}\right)=\tilde{f}_{g}^{C C F M}\left(x, b, \mu^{2}\right) \cdot F_{g}^{n p}(b) .
$$

The form factor responsible for the nonperturbative effects must be normalized such that

$$
F_{g}^{N P}(b=0)=1
$$

Then by construction:

$$
\tilde{f}_{g}\left(x, b=0, \mu^{2}\right)=\frac{x}{2} g\left(x, \mu^{2}\right) .
$$

In the following, for simplicity, we use an $x$-independent form factor

$$
F_{g}^{n p}(b)=\exp \left(-\frac{b^{2}}{4 b_{0}^{2}}\right)
$$

which is responsible for the nonperturbative effects. The Gaussian form factor in $b$ means also a Gaussian initial momentum distribution $\exp \left(-k_{t}^{2} b_{0}^{2}\right)$ (Fourier transform of a Gaussian function is a Gaussian function). Gaussian form factor is often used to correct collinear pQCD calculations for so-called intrinsic momenta. Other functional forms in $b$ are also possible.

The similarities and differences betwen the standard soft gluon resummation and the CCFM resummation have been discussed recently in Ref. [17]. It has been shown how the soft gluon resummation formulae can be obtained as the result of the approximate treatment of the solution of the CCFM equation in the so-called b-representation.

\subsubsection{Off-shell matrix element for $g^{*} g^{*} \rightarrow H$}

While in the collinear approach the off-shell matrix elements are needed only at higher orders [19], in the $k_{t}$-factorization approach the off-shell matrix elements appear in principle already at the leading order. In Ref. [19] the matrix element was calculated in the framework of the effective Lagrangian for the 
Higgs boson coupling to gluons in the infinitely heavy top mass approximation. While the on-mass-shell couplings in the full theory and in the effective Lagrangian theory are equivalent, this is not expected for matrix element with off-shell gluons. In particular the dependence on transverse momenta and their relative orientation can be different. The use of the effective vertex all over the phase space may not be completely realistic. However, the matrix element in the full theory (with finite top mass) was not yet calculated.

In the present paper we shall only estimate the off-shell effects in the effective Lagrangian approximation. Then the cross section for the Higgs production can be written as:

$$
\frac{d \sigma^{H}}{d y d^{2} p_{t}}=\frac{\sigma_{0}^{g g \rightarrow H}}{(2 \pi)^{2}} \int f_{g / 1}\left(x_{1}, \kappa_{1}^{2}, \mu^{2}\right) f_{g / 2}\left(x_{2}, \kappa_{2}^{2}, \mu^{2}\right) 2 \frac{\left(\vec{\kappa}_{1} \cdot \vec{\kappa}_{2}\right)^{2}}{\kappa_{1}^{2} \kappa_{2}^{2}} \lambda\left(\kappa_{1}^{2}, \kappa_{2}^{2}, p_{t}^{2}\right) d^{2} q_{t},
$$

where $\lambda$ is a smooth function of its parameters [19, 20. In the limit $p_{t}^{2} \rightarrow 0$, $\kappa_{1}^{2} \rightarrow 0, \kappa_{2}^{2} \rightarrow 0$ the dimensionless function $\lambda\left(\kappa_{1}^{2}, \kappa_{2}^{2}, p_{t}^{2}\right) \rightarrow 1$. Then the 2 $\cos ^{2} \phi_{\vec{\kappa}_{1}, \vec{\kappa}_{2}}$ factor in the formula above constitute the essential difference with respect to the on-shell approximation. It modifies the dependence of the integrand under the $\phi_{q}$ integration.

In Fig 2 we show the ratio:

$$
R=\frac{\left(d \sigma / d p_{t}\right)_{\text {off-shell }}}{\left(d \sigma / d p_{t}\right)_{\text {on-shell }}}
$$

as a function of Higgs-boson transverse momentum. In this calculation the BFKL unintegrated gluon distributions have been used as an example. The result for other distributions is similar. The final effect is rather small (less than $10 \%$ ) in the region of our interest and depends on the way how the flux factor for off-shell gluons is defined [19, 20] ${ }^{5}$. At finite, but not too large, Higgs boson transverse momenta the averaging over gluon transverse momenta with UGDF's gives $<2 \cos ^{2} \phi_{\kappa_{1}, \kappa_{2}}>\approx 1$, and one approximately recovers the on-shell result. This is not true for $p_{t} \approx 0$, when $\overrightarrow{\kappa_{1}}$ and $\overrightarrow{\kappa_{2}}$ are strongly anticorrelated and the averaging is not efficient. The off-shell effect on the integrated cross section $(d \sigma / d y)$ is even smaller. Here we wish to concentrate rather on the effect of transverse momenta inherent for UGDF's. We shall leave a detailed study of the off-shell effects in effective Lagrangian and in the full theory for the future and consequently we shall use the onshell matrix element in the following. This approximation will be also useful here when comparing the $k_{t}$-factorization results with that for the standard

\footnotetext{
${ }^{5}$ While it is rather strightforward to calculate matrix element for off-shell gluons, there is some ambiguity in defining the flux factor for virtual gluons.
} 
collinear and soft-gluon resummation approaches. The on-shell approximation was used recently in the formalism od doubly unintegrated parton distribution for electroweak boson production [18.

\subsubsection{Standard soft-gluon resummation}

The formula for inclusive cross section in terms of unintegrated gluon distributions in the impact parameter space looks very similar to the one in the standard soft gluon resummation approach known from the literature. This similarity is not random [17. In the Collins-Soper-Sterman (CSS) approach [16] the resummed cross section for Higgs production reads

$$
\begin{array}{r}
\frac{d \sigma}{d y d^{2} p_{t, H}}=\frac{\sigma_{0}^{g g \rightarrow H}}{(2 \pi)^{2}} \int d^{2} b J_{0}\left(p_{t} b\right) W_{g g}^{N P}\left(b, x_{1}, x_{2}, \mu^{2}\right) \\
x_{1} \cdot\left[g_{1}\left(x_{1}, \mu(b)\right)+\frac{\alpha_{s}(\mu(b))}{2 \pi} C_{v c} g_{1}\left(x_{1}, \mu(b)\right)+\frac{\alpha_{s}(\mu(b))}{2 \pi} \sum_{f_{1}}\left(C_{g q} \otimes q_{1}^{f_{1}}\right)\left(x_{1}, \mu(b)\right)\right] \\
x_{2} \cdot\left[g_{2}\left(x_{2}, \mu(b)\right)+\frac{\alpha_{s}(\mu(b))}{2 \pi} C_{v c} g_{2}\left(x_{2}, \mu(b)\right)+\frac{\alpha_{s}(\mu(b))}{2 \pi} \sum_{f_{2}}\left(C_{g q} \otimes q_{2}^{f_{2}}\right)\left(x_{2}, \mu(b)\right)\right] \\
\exp \left[\frac{1}{2}\left(S_{g}\left(b, \mu^{2}\right)+S_{g}\left(b, \mu^{2}\right)\right)\right],
\end{array}
$$

where the exponents in the Sudakov-like form factors read

$$
S_{g}\left(b, \mu^{2}\right)=-\int_{\bar{\mu}_{\text {min }}^{2}(b)}^{\mu^{2}} \frac{d \bar{\mu}^{2}}{\bar{\mu}^{2}}\left[\ln \left(\frac{\mu^{2}}{\bar{\mu}^{2}}\right) A_{g}\left(\alpha_{s}\left(\bar{\mu}^{2}\right)\right)+B_{g}\left(\alpha_{s}\left(\bar{\mu}^{2}\right)\right)\right] .
$$

The coefficient functions $C$ 's in Eq.(16) can be found in 21]. The coefficient $A$ and $B$ in the Sudakov-like form factor can be expanded in the series of $\alpha_{s}$ :

$$
\begin{aligned}
& A_{g}=2 C_{A} \frac{\alpha_{s}(\bar{\mu})}{2 \pi}+\left(\frac{\alpha_{s}(\bar{\mu})}{2 \pi}\right)^{2}(\ldots)+\ldots, \\
& B_{g}=-2 \beta_{0} \frac{\alpha_{s}(\bar{\mu})}{2 \pi}+\left(\frac{\alpha_{s}(\bar{\mu})}{2 \pi}\right)^{2}(\ldots)+\ldots,
\end{aligned}
$$

where $\beta_{0}=\frac{11}{6} C_{A}-\frac{2}{3} N_{F} T_{R}\left(T_{R}=\frac{1}{2}, N_{F}=5, C_{A}=3\right)$. The CSS formalism [16. leaves open the question of small $b$. Different prescriptions have been proposed to treat this region. The lower limit of the integral in Eq.(17) is usually taken $\mu_{\text {min }}^{2}(b)=\left(\frac{C_{b}}{b}\right)^{2}$, where $C_{b}=2 \exp \left(-\gamma_{E}\right) \approx 1.1229$. This prescription leads to a kink for the Sudakov form factor if $C_{b} / b=\mu$. To allow 
a smooth dependence and to quarantee that the lower limit is really lower than the upper limit, one could make the following replacement $\mu_{\min }^{2}(b)=$ $\left(\frac{C_{b}}{b}\right)^{2} \rightarrow\left(\frac{C_{b}}{b}\right)^{2}\left[1+C_{b}^{2} /\left(b^{2} \mu^{2}\right)\right]^{-1}$. To quarantee that the scale of parton distribution does not take unphysically small value we shall use the following prescription:

$$
\mu^{2}(b)=\mu_{\min }^{2}(b)+\mu_{0}^{2},
$$

where $\mu_{0}^{2}$ is the starting value for the QCD evolution. In the present paper we shall use easy to handle leading order parton distributions from Ref. [28].

$W_{g g}^{N P}\left(b, x_{1}, x_{2}, \mu^{2}\right)$ in Eq.(16) is of nonperturbative origin. Different effective parametrizations have been proposed in the literature. Assuming a factorizable form of the $W_{g g}^{N P}$ function

$$
W_{g g}^{N P}\left(b, x_{1}, x_{2}, \mu^{2}\right)=F_{g}^{N P}\left(b, x_{1}, \mu^{2}\right) \cdot F_{g}^{N P}\left(b, x_{2}, \mu^{2}\right)
$$

the soft-gluon-resummation formula (16) and the unintegrated gluon distribution formula (8) for Higgs production in the b space have identical structure if the following formal assignment is made:

$$
\tilde{f}_{g}^{S G R}\left(x, b, \mu^{2}\right)=\frac{1}{2} F_{g}^{N P}\left(b, x, \mu^{2}\right)\left[x g\left(x, \mu^{2}(b)\right)+\ldots\right] \exp \left(\frac{1}{2} S_{g}\left(b, \mu^{2}\right)\right) \text {. }
$$

If the off-shell matrix element for $g g \rightarrow H$ is taken in the UGDF approach with the Kwieciński distribution, the structure of the formula in both approaches would be different. In this sense the UGDF approach seems more general than the b-space resummation method.

\section{$2.2 \quad 2 \rightarrow 2$ processes}

At sufficiently large transverse momenta $\left(p_{t}>M_{H}\right)$ the Higgs boson production of the type $2 \rightarrow 2$ should dominate over the $2 \rightarrow 1$ mechanism discussed above. The cross section for fixed-order processes of the type $p_{1} p_{2} \rightarrow H p_{3}$ 
(parton+parton $\rightarrow$ Higgs + parton) of the order of $\alpha_{s}$ is well known [31]

$$
\begin{aligned}
\frac{d \sigma}{d y_{H} d y_{p} d^{2} p_{t}}\left(y_{W}, y_{p}, p_{t}\right) & =\frac{1}{16 \pi^{2} \hat{s}^{2}} \\
& \times\left\{x_{1} g_{1}\left(x_{1}, \mu^{2}\right) x_{2} g_{2}\left(x_{2}, \mu^{2}\right) \overline{\left|\mathcal{M}_{g g \rightarrow H g}\right|^{2}}\right. \\
& +\left[\sum_{f_{1}=-3,3} x_{1} q_{1, f_{1}}\left(x_{1}, \mu^{2}\right)\right] x_{2} g_{2}\left(x_{2}, \mu^{2}\right) \overline{\left|\mathcal{M}_{q g \rightarrow H q}\right|^{2}} \\
& +x_{1} g_{1}\left(x_{1}, \mu^{2}\right)\left[\sum_{f_{2}=-3,3} x_{2} q_{2, f_{2}}\left(x_{2}, \mu^{2}\right)\right] \overline{\left|\mathcal{M}_{g q \rightarrow H q}\right|^{2}} \\
& \left.+\sum_{f=-3,3} x_{1} q_{1, f}\left(x_{1}, \mu^{2}\right) x_{2} q_{2,-f}\left(x_{2}, \mu^{2}\right) \overline{\left|\mathcal{M}_{q q \rightarrow H g}\right|^{2}}\right\} .
\end{aligned}
$$

The indices $f$ in the formula above number both quarks $(f>0)$ and antiquarks $(f<0)$. Only three light flavours are included in actual calculations. The explicit formulae for $\overline{|\mathcal{M}|^{2}}$ can be found in 31 .

\subsection{Weak boson fusion}

Up to now we have discussed only the contribution of the dominant LO gluon-gluon fusion and NLO $2 \rightarrow 2$ corrections and completely ignored contributions of other processes. The second most important mechanism for Higgs production is the fusion of off-shell gauge bosons: WW or ZZ. It is known that at LHC energy and intermediate mass $\left(100 \mathrm{GeV}<M_{H}<500\right.$ $\mathrm{GeV}$ ) Higgs the WW fusion constitutes about 10-15\% of the integrated inclusive cross section. If the weak boson fusion contribution was separated, the measurement of the WWH (or ZZH) coupling would be very interesting test of the Standard Model.

Previous studies of the WW mechanism concentrated on the total cross section for the Higgs production. In the present paper we are interested in

differential distributions of Higgs boson rather than in the integrated cross section.

For the gauge boson fusion the partonic subprocess is of the $2 \rightarrow 3$ type: $q\left(p_{1}\right)+q\left(p_{2}\right) \rightarrow q\left(p_{3}\right)+q\left(p_{4}\right)+H\left(p_{H}\right)$. The corresponding hadronic cross section can be written as

$$
\begin{array}{r}
d \sigma=\mathcal{F}_{12}^{V V}\left(x_{1}, x_{2}\right) \frac{1}{2 \hat{s}} \overline{\left|\mathcal{M}_{q q \rightarrow q q H}\right|^{2}} \frac{d^{3} p_{3}}{(2 \pi)^{3} 2 E_{3}} \frac{d^{3} p_{4}}{(2 \pi)^{3} 2 E_{4}} \frac{d^{3} p_{H}}{(2 \pi)^{3} 2 E_{H}} \\
(2 \pi)^{4} \delta^{4}\left(p_{1}+p_{2}-p_{3}-p_{4}-p_{H}\right) d x_{1} d x_{2} .
\end{array}
$$


The next-to-leading order corrections to the matrix element of the WW fusion are rather small 32]. For comparison the NLO corrections for gluon-gluon fusion are significantly larger. Since we wish to concentrate on relative effect of the gluon-gluon and WW fusion contributions in the following we restrict to a much simpler leading order (LO) calculation. The LO subprocess matrix element was calculated first in Ref. 33]. The spin averaged matrix element squared reads

$$
\overline{|\mathcal{M}|^{2}}=128 \sqrt{2} G_{F}^{3} \frac{M_{W}^{8}\left(p_{1} \cdot p_{2}\right)\left(p_{3} \cdot p_{4}\right)}{\left(2 p_{3} \cdot p_{1}+M_{W}^{2}\right)^{2}\left(2 p_{4} \cdot p_{2}+M_{W}^{2}\right)^{2}} .
$$

For the WW fusion, limiting to light flavours, the partonic function is

$$
\begin{array}{r}
\mathcal{F}_{12}^{W W}\left(x_{1}, x_{2}\right)= \\
\left(u_{1}\left(x_{1}, \mu_{1}^{2}\right)+\bar{d}_{1}\left(x_{1}, \mu_{1}^{2}\right)+\bar{s}_{1}\left(x_{1}, \mu_{1}^{2}\right)\right)\left(\bar{u}_{2}\left(x_{2}, \mu_{2}^{2}\right)+d_{2}\left(x_{2}, \mu_{2}^{2}\right)+s_{2}\left(x_{2}, \mu_{2}^{2}\right)\right)+ \\
\left(\bar{u}_{1}\left(x_{1}, \mu_{1}^{2}\right)+d_{1}\left(x_{1}, \mu_{1}^{2}\right)+s_{1}\left(x_{1}, \mu_{1}^{2}\right)\right)\left(u_{2}\left(x_{2}, \mu_{2}^{2}\right)+\bar{d}_{2}\left(x_{2}, \mu_{2}^{2}\right)+\bar{s}_{2}\left(x_{2}, \mu_{2}^{2}\right)\right) .
\end{array}
$$

We take either (i) $\mu_{1}^{2}=\mu_{2}^{2}=M_{H}^{2}$ or (ii) $\mu_{1}^{2}=-t_{1}, \mu_{2}^{2}=-t_{2}$, where $t_{1}$ and $t_{2}$ are virtualities of $\mathrm{W}$ bosons. It is convenient to introduce the following new variables:

$$
\begin{aligned}
& \vec{p}_{+}=\vec{p}_{3}+\vec{p}_{4}, \\
& \vec{p}_{-}=\vec{p}_{3}-\vec{p}_{4},
\end{aligned}
$$

which allow to eliminate the momentum-dependent $\delta^{3}(\ldots)$ in Eq.(23). Instead of integrating over $x_{1}$ and $x_{2}$ we shall integrate over $y_{1} \equiv \ln \left(1 / x_{1}\right)$ and $y_{2} \equiv \ln \left(1 / x_{2}\right)$. Then using Eq.(23) we can write the inclusive spectrum of Higgs as

$$
\begin{aligned}
\frac{d \sigma}{d y d^{2} p_{t}}= & \int d y_{1} d y_{2} x_{1} x_{2} \mathcal{F}\left(x_{1}, x_{2}, \mu_{1}^{2}, \mu_{2}^{2}\right) \frac{1}{2 \hat{s}} \frac{d^{3} p_{-}}{16} \overline{\left|\mathcal{M}_{q q \rightarrow q q H}\right|^{2}} \frac{1}{2 E_{3}} \frac{1}{2 E_{4}} \\
& \frac{1}{(2 \pi)^{5}} \delta\left(E_{1}+E_{2}-E_{3}-E_{4}-E_{H}\right) .
\end{aligned}
$$

This is effectively a four-dimensional integral which can be easily calculated numerically.

\section{Results}

\subsection{Gluon-gluon fusion}

Since we wish to concentrate on the potential to verify different UGDF's rather than to present the best predictions for LHC experiments we shall 
consider only one mass of the Higgs boson $M_{H}=125 \mathrm{GeV}$ as an example. This is slightly above the lower limit obtained from the analysis of the LEP data [34. In addition, this is a mass for which many calculations in the literature has been performed recently. Therefore this gives a chance of a comparison to the existing results.

Before we go to the analysis of the two-dimensional spectra of Higgs boson produced in proton-proton or proton-antiproton collisions let us show the range of gluon longitudinal momentum fraction tested in these proceses. In Fig 3 we present correspondingly $x_{1}$ and $x_{2}$ as a function of Higgs boson rapidity for a few different values of Higgs transverse momentum. While at Tevatron energies (panel (a)) only intermediate and large $x$ 's come into the game, in collisions at LHC energy (panel (b)) $\mathrm{x} \sim 10^{-2}$ is sampled at midrapidity. However, at LHC energy, at rapidities $|y|>2$ one enters the region of $x>0.1$. Here some of the low-x models of UGDF's may become invalid.

Let us concentrate first on transverse momentum distributions. The distribution of Higgs transverse momentum (rapidity integrated) is shown in Fig 4. In Fig [5] we present transverse momentum distribution of Higgs boson in different bins of rapidity specified in the figure caption. At midrapidity (panel (a)) only small $x$ 's are sampled. Even here different models from the literature give quite different transverse momentum distributions although all of them give a reasonable description of the HERA data. The LO soft-gluon resummation distribution and the distribution obtained with the Kwieciński unintegrated gluon distribution $\left(b_{0}=1 \mathrm{GeV}^{-1}\right)$ are very similar with maxima at $p_{t, H} \approx 10 \mathrm{GeV}$ and $p_{t, H} \approx 5 \mathrm{GeV}$, respectively. The cross section for $\mathrm{W}$ or $\mathrm{Z}$ production is fairly sensitive to the choice of the nonperturbative form factor [12. In contrast to the gauge boson production the Higgs boson production is much less sensitive to the parameter of the Gaussian form factor. The results with $b_{0}=0.5$ or $2 \mathrm{GeV}^{-1}$ (not shown here) almost coincide with the result for $b_{0}=1 \mathrm{GeV}^{-1}$. Therefore in the following in all calculations we shall use $b_{0}=1 \mathrm{GeV}^{-1}$. The BFKL type gluon distributions lead to much larger cross section at small Higgs transverse momenta and a sizeably larger slope of the $p_{t}$-distribution. The rapid fall-off of the cross section for the Golec-Biernat-Wüsthoff nonperturbative gluon distribution [24] (thin dashed curve) demonstrates how important is the perturbative initial state radiation in generating larger transverse momenta of the Higgs boson. Such effects are not taken into account in Ref. [24].

A comment regarding the GBW distribution is here in order. This distribution was obtained based on a simple dipole parametrization of the HERA data inspired by the saturation idea. In addition to the very steep distribution in Higgs-boson transverse momentum the scale-independent GBW gluon 
distribution gives very small total cross section (about half of $1 \mathrm{pb}$ ). One has to remember, however, that this distribution was constructed in order to describe $\sigma_{\gamma^{*} p}^{t o t}\left(Q^{2}\right)$ for small photon virtualities. We wish to stress here that this distribution is not an universal object. For example in its simplest form it fails to describe $\sigma_{\gamma^{*} p}^{t o t}\left(Q^{2}\right)$ for large photon virtualities. The corresponding effective gluon distribution defined as

$$
x g_{G B W}(x) \equiv \int_{0}^{\infty} \mathcal{F}_{g}^{G B W}(x) d \kappa^{2}
$$

resembles the standard collinear distribution $x g_{D G L A P}\left(x, \mu^{2}\right)$ for small factorization scale $\mu^{2} \sim 1 \mathrm{GeV}^{2}$. The latter, when substituted into the standard leading-order formula, also leads to a small total cross section of the order of $1 \mathrm{pb}$. A reasonable total cross section is obtained provided $\mu^{2} \sim M_{H}^{2}$. Although $g_{D G L A P}\left(x, \mu^{2}=1 G e V^{2}\right)$ leads to a reasonable description of $F_{2}$ at $Q^{2} \sim 1 \mathrm{GeV}^{2}$ it cannot be directly (without DGLAP evolution) used for Higgs production. In this context the scale-independent GBW distribution should be understood as an initial condition for QCD evolution rather than an universal object to be used in different high-energy processes.

Different UGDF's constructed in order to describe the total cross section for $\gamma^{*} p$ process give quite different predictions for the Higgs production. This, as will be discussed below, is not completely surprising. While the $\gamma^{*} p$ total cross section is sensitive to the integral $\int d k_{t}^{2} f_{g}\left(x, k_{t}^{2}\left(, \mu^{2}\right)\right)$, the Higgs boson transverse momentum distribution samples details of the unintegrated gluon distributions. As discussed in the previous section, in the case of Higgs boson production the inclusive cross section is a convolution of two UGDF's. In general, exclusive reactions are much better place for testing UGDF's. In this sense the standard procedure to constrain UGDF's through describing the $F_{2}$ HERA data does not seem very effective.

In order to understand the situation somewhat better in Fig [6 we show the corresponding average values of sampled transverse momenta as the function of Higgs transverse momentum. At $\mathrm{y}=0$, by symmetry requirement, $\left\langle\kappa_{1}\right\rangle$ and $\left\langle\kappa_{2}>\right.$ are identical. It is not the case for $\mathrm{y}=3$. Rather similar results are obtained with different UGDF's. At very small Higgs transverse momenta one tests $\kappa$ 's $\sim 1 \mathrm{GeV}$. At large Higgs transverse momenta and $\mathrm{y}=0$ we get $<\kappa_{1}>+<\kappa_{2}>\approx p_{t} / 2$. Of course by symmetry $<\kappa_{1 / 2}>(-y)=<\kappa_{2 / 1}>$ $(y)$.

Some examples of inclusive rapidity distributions are shown in Fig:7. Even here the differences between different UGDF's are clearly visible. Above $|y|>3$ only resummation distribution (thin solid line), Kwieciński distribution (thick solid line) and Kimber-Martin-Ryskin (dotted line) are applicable 
by construction. The other distributions were obtained by extending the generally small-x gluon distributions above $\mathrm{x}>0.1$ by multiplying the original formulae by $(1-x)^{n}$. The power $\mathrm{n}=5-7$ was found recently in the production of $c \bar{c}$ pairs in photon-proton scattering at low energies 4. In the present paper we used $n=7$. Small differences may be expected only at the very edges of the phase space, i.e. in the region we are not interested here.

For completeness in Fig 8 we show the average values of gluon transverse momenta $\left\langle\kappa_{1}>\right.$ and $\left\langle\kappa_{2}>\right.$ as a function of Higgs-boson rapidity. At midrapidity by symmetry $<\kappa_{1}>\approx<\kappa_{2}>$. The average values strongly depend on UGDF used and on the region of rapidity. The asymmetry of average values of the transverse momenta at forward/backward regions are closely related to Fig [3 due to correlation of transverse momenta with longitudinal momentum fractions. Generally, the smaller $x_{1}\left(x_{2}\right)$ the larger $<\kappa_{1}>$ $\left(<\kappa_{2}>\right)$. The details depend, however, on the specific version of the gluon dynamics. The extremely small average transverse momenta for the GBW UGDF can be understood in the light of the discussion above.

How important is the choice of the factorization scale in our calculations with Kwieciński UGDF? In Fig 9 we show results obtained with quite different choices of factorization scale and with $b_{0}=1 \mathrm{GeV}^{-1}$. There is very little effect if the factorization scale is increased from our cannonical value $\mu^{2}=M_{H}^{2}$. There is, however, a sizeable effect if the factorization scale is decreased drastically, especially at $p_{t}>50 \mathrm{GeV}$. The factorization scale dependence in our case seems somewhat weaker than in a recent work [20].

It is particularly interesting to compare the results obtained with the Kwieciński unintegrated gluon distributions with those obtained from the standard soft-gluon resummation method. In Fig[10] we show two-dimensional distributions in $\left(y, p_{t}\right)$. The distribution obtained with the Kwieciński UGDF decrease less rapidly with Higgs transverse momentum than the distribution obtained in the standard soft-gluon resummation. This is partially due to the different choice of the factorization scale in both methods.

A more detailed comparison is made in Fig 11 where we have selected two rapidities $y=0$ and $y=3$. While at $\mathrm{y}=0$ the result obtained with Kwieciński distributions and that obtained within the standard resummation method almost coincide, they become quite different at $\mathrm{y}=3$ and $p_{t}>$ $60 \mathrm{GeV}$. However, the applicability of both methods at large transverse momenta is not obvious. The result for $\mathrm{y}=3$ at large transverse momenta obtained with Kwieciński UGDF seems more trustworthy than that obtained within standard resummation method. The problems of the standard resummation method at large transverse momenta may be caused by some somewhat arbitrary prescriptions used as discussed in section 2 .

In order to better emphasize the differences between different UGDF's we 
separate the contributions to integral in Eq.(6) from four different disjoint and complementary kinematic regions:

- (I) $\kappa_{1}<p_{t}$ and $\kappa_{2}<p_{t}$

- (II) $\kappa_{1}<p_{t}$ and $\kappa_{2}>p_{t}$,

- (III) $\kappa_{1}>p_{t}$ and $\kappa_{2}<p_{t}$,

- (IV) $\kappa_{1}>p_{t}$ and $\kappa_{2}>p_{t}$

where $\kappa_{1}$ and $\kappa_{2}$ are transverse momenta of the last gluons in the ladders and $p_{t}$ is transverse momentum of the produced Higgs boson. In Fig 12 we present the decomposition of the Higgs cross section $\frac{d \sigma}{d p_{t}}$ into those four regions as a function of Higgs transverse momentum. Here we limit to midrapidities (-1 $<y<1$ ) only. In the case of Kwieciński UGDF, first the Fourier transform from the b-space to $\kappa_{t}$-space was calculated and the results were stored on the grid in $\mathrm{x}$ and $\kappa_{t}^{2}$. The grid was used then for interpolation when using formula (6) with the extra conditions on transverse momenta specified above. It is interesting to note that at larger transverse momenta the contributions from regions II, III and IV are completely negligible. The other contributions are important only at very low transverse momenta. For completeness in Fig 13 we show similar decomposition as a function of rapidity. In this calculation we have limited Higgs transverse momenta to $p_{t}<40 \mathrm{GeV}$. The contribution of region I dominates at midrapidities. The contribution of asymmetric (in $\kappa_{1}$ and $\kappa_{2}$ ) regions II and III becomes important at very forward or very backward Higgs production. The contribution of the region IV is negligible almost everywhere. The proportions of contributions corresponding to the four specified above regions differ significantly for different UGDF's.

\subsection{Estimates of higher-order effects}

In the present paper we have limited to leading-order approach only. This was dictated by the fact that until now only leading-order approach was used to describe the HERA data in terms of UGDF's. Furthermore the consistent next-to-leading order analysis is rather complicated. We leave the next-to-leading order analysis for a future study. Instead, we wish to visualize (estimate) the NLO corrections in a similar soft-gluon-resummation approach where the relevant formalism was worked out in detail [21. In Fig[14 we present the relevant soft-gluon-resummation K-factor as a function of Higgs transverse momentum for $-0.5<y<0.5$ (panel a) and $2.5<y<3.5$ (panel b). In this calculation the Gaussian form factor (see Eq.(13)) with $b_{0}=1$ 
$\mathrm{GeV}^{-1}$ was used. The dashed line includes only gluonic NLO corrections to partonic function, the dotted line exclusively the quark NLO corrections and the solid line includes all NLO effects altogether. At midrapidities the gluonic effects are absolutely dominant and enhance the leading-order cross section by about $80 \%$. The quark corrections are at the level of $1 \%$ and can be numerically neglected. They become sizable (of the order of $10 \%$ ) at very forward and very backward rapidities. In Fig [15 we present a corresponding $\mathrm{K}$-factor $(\mathrm{K}=\mathrm{NLO} / \mathrm{LO})$ as a function of Higgs rapidity. In this calculations $p_{t, H}<80 \mathrm{GeV}$ was assumed. Summarizing, to a good approximation the NLO soft-gluon resummation corrections result in multiplying the LO cross section for Higgs production by a factor of about 1.8 .

Up to now we have concentrated at relatively small Higgs transverse momenta. At high transverse momenta the standard $2 \rightarrow 2$ mechanisms take over. The cross section for perturbative $2 \rightarrow 2$ processes with Higgs in the final state is shown in Fig [16. The $g g \rightarrow H g$ dominates at small transverse momenta and midrapidities. The $q g \rightarrow H q$ and $g q \rightarrow H q$ become comparable to the first contribution at large transverse momenta and forward and backward regions, respectively. The contribution of $q q \rightarrow H g$ is negligible all over the interesting part of the phase space.

\subsection{Weak boson fusion versus gluon-gluon fusion}

The weak boson fusion is known to be another important ingredient in total (integrated) cross section for Higgs boson production [14. It is interesting what is interrelation between the two dominant contributions in rapidity and transverse momentum of the Higgs boson. In Fig 17 we present such a twodimensional spectrum. This spectrum is very different from those for the gluon-gluon fusion. In particular, the maximum of the cross section at $p_{t}$ slightly larger than $50 \mathrm{GeV}$ is visible. It is interesting if the contribution of weak-boson fusion can exceed in some corner of the phase space the gluongluon contribution. In order to quantify the effect in Fig 18 we present $\frac{d \sigma}{d y d p_{t}}$ as a function of Higgs transverse momentum for $y=0$ and $y= \pm 3$. The contribution of the WW fusion falls off much faster for $y= \pm 3$ than for $y=0$. The results are almost independent of the choice of the factorization scale. These results almost coincide (compare thick solid (prescription (i) above) and overlaping thick dashed (prescription (ii) above) lines). For comparison we present a few examples of the gluon-gluon fusion with BFKL (dash-dotted) and Kwieciński (thin solid) unintegrated gluon distributions and standard resummation method with Gaussian form factor and $b_{0}=1$ $\mathrm{GeV}^{-1}$ (dashed). We find that depending slightly on UGDF and rapidity, above $p_{t} \sim 50-100 \mathrm{GeV}$ the WW fusion mechanism dominates over gluon- 
gluon fusion mechanism. However, the $2 \rightarrow 2$ processes (dotted line) are large, especially at midrapidities. Only at large rapidities and $p_{t}>150 \mathrm{GeV}$ the WW fusion seems to dominate over the other processes. However, there the cross section is rather small. Whether this opens a possibility to study the WWH and similarly ZZH couplings requires further studies.

\section{Conclusions}

In the present paper we have presented predictions for the inclusive cross section for the Higgs boson production at the LHC energy $\mathrm{W}=14 \mathrm{TeV}$, obtained with the help of different models of unintegrated gluon distributions used recently in the literature. Almost all the UGDF's discussed here were obtained based on the analysis of low-x HERA data for virtual photon - proton total cross sections. Although they are almost equivalent in the description of the HERA data, quite different results have been obtained for Higgs production. While the structure function data is sensitive to rather low transverse momenta of the gluon, in the Higgs production, in principle, one could sample not yet explored region of large transverse momenta (at large scales). One should remember that the Higgs production even at large LHC energy is not completely a small-x phenomenon. The analysis of very forward or very backward Higgs boson production, in principle, could open a possibility to study UGDF's in a completely unexplored region of large x. This task, however, is by no means easy as in this region of phase space both "small-x" and "large-x" physics is intertangled.

We have shown that for all UGDF's discussed here the inclusive cross section is dominated by the configurations with transverse momenta of the gluons smaller than the transverse momentum of the Higgs $\left(\kappa_{1}<p_{t}\right.$ and $\left.\kappa_{2}<p_{t}\right)$.

Finally we wish to emphasize that all the potential to study UGDF in the Higgs production discussed here is at present only conditional as it implicitly assumes the existence, discovery and good identification of the Higgs boson in the future experiments at LHC. We do not need to mention that all this would not be possible if the Higgsless scenarios (see e.g. 35] and references therein) turned out to be true. Even if the Higgs boson is discovered at LHC the statistics may not be sufficient for precise tests of UGDF.

Acknowledgements We are indebted to Krzysztof Golec-Biernat, Elżbieta Richter-Wąs and Tadeusz Szymocha for an interesting discussion and Krzysztof Kutak for providing us a routine for calculating unintegrated gluon distributions from Ref. [26]. The paper has been improved thanks to suggestions of 
an anonymous referee. This work was partially supported by the grant of the Polish Ministry of Scientific Research and Information Technology number 1 P03B 02828.

\section{References}

[1] Bo Anderson et al. (Small-x collaboration), Eur. Phys. J. C25 (2002) 77.

[2] J. Andersen et al. (Small-x collaboration), Eur. Phys. J. C35 (2004) 67.

[3] A. Szczurek, N.N. Nikolaev, W. Schäfer and J. Speth, Phys. Lett. B500 (2001) 254.

[4] M. Łuszczak and A. Szczurek, Phys. Lett. B B59 (2004) 291.

[5] H. Jung, Mod. Phys. Lett. A19 (2004) 1.

[6] A. Szczurek, Acta Phys. Polon. B34 (2003) 3191.

[7] D. Kharzeev and E. Levin, Phys. Lett. B523 (2001) 79.

[8] M.A. Kimber and A.D. Martin and M.G. Ryskin, Phys. Rev. D63 (2001) 114027-1.

[9] J. Kwieciński, Acta Phys. Polon. B33 (2002) 1809.

[10] A. Gawron and J. Kwieciński, Acta Phys. Polon. B34 (2003) 133.

[11] A. Gawron, J. Kwieciński and W. Broniowski, Phys. Rev. D68 (2003) 054001.

[12] J. Kwieciński and A. Szczurek, Nucl. Phys. B680 (2004) 164.

[13] M. Czech and A. Szczurek, Phys. Rev. C72 (2005) 015202;

M. Czech and A. Szczurek, nucl-th/0510007.

[14] R.K. Ellis, W.J. Stirling and B.R. Webber, QCD and Collider Physics, Cambridge University Press, Cambridge 1996.

[15] M. Ciafaloni, Nucl. Phys. B296 (1988) 49;

S. Catani, F. Fiorani and G. Marchesini, Phys.Lett.B234 (1990) 339;

Nucl. Phys. B336 (1990) 18. 
[16] J.C. Collins, D. Soper and G. Sterman,

Phys. Lett. B109 (109) (1982) 388;

Nucl. Phys. B223 (1983) 381;

Phys. Lett. B126 (1983) 275;

Nucl. Phys. B250 (1985) 199.

[17] A. Gawron and J. Kwieciński, Phys. Rev. D70 (2004) 014003.

[18] G. Watt, A.D. Martin and M.G. Ryskin, Phys. Rev. D70 (2004) 014012.

[19] F. Hautmann, Phys. Lett. B535 (2002) 159.

[20] A.V. Lipatov and N.P. Zotov, hep-ph/0501172.

[21] I. Hinchliffe and S.F. Novaes, Phys. Rev. D38 (1988) 3475;

R.P. Kauffman, Phys. Rev. D44 (1991) 1415;

C.-P. Yuan, Phys. Lett. B283 (1992) 395;

C. Balazs and C.P. Yuan, Phys. Lett. B478 (2000) 192;

C. Balazs, J. Huston and I. Puljak, Phys. Rev. D63 (2001) 014021;

E.L. Berger and J.-W. Qiu, Phys. Rev. D67 (2003) 034026;

G. Bozzi, S. Catani, D. de Florian and M. Grazzini, Phys. Lett. B564 (2003) 65.

[22] A. Kulesza and W.J. Stirling, JHEP 0312 (2003) 056.

[23] A. Kulesza, G. Sterman and W. Vogelsang, hep-ph/0309264.

[24] K. Golec-Biernat and M. Wüsthoff, Phys. Rev. D60 (1999) 114023.

[25] A.J. Askew, J. Kwieciński, A.D. Martin and P.J. Sutton, Phys. Rev. D49 (1994) 4402.

[26] K. Kutak and A.M. Staśto, Eur. Phys. J. C41 (2005) 343.

[27] J. Blümlein, a talk at the workshop on Deep Inelastic Scattering and QCD, hep-ph/9506403.

[28] M. Glück, E. Reya and A. Vogt, Eur. Phys. J. C5 (1998) 461.

[29] J.R. Ellis, M.K. Gaillard and D.V. Nanopoulos, Nucl. Phys. B106 (1976) 292.

[30] V. Del Duca, W. Kilgore, C. Oleari, C. Schmidt and D. Zeppenfeld, Phys. Rev. D67 (2003) 073003. 
[31] R.K. Ellis, I. Hinchliffe, M. Soldate and J.J. van der Bij, Nucl. Phys. B297 (1988) 221;

U. Baur and E.W.N. Glover, Nucl. Phys. B339 (1990) 38.

[32] T. Figy, D. Zeppenfeld and C. Oleari, Phys. Rev. D68 (2003) 073005.

[33] R.N. Cahn and S. Dawson, Phys. Lett. B136 (1984) 196.

[34] ALEPH, DELPHI, L3, OPAL collaborations, LEP Electroweak Working Group and SLD Electroweak, Heavy Flavour Groups, hep-ex/0412015.

[35] R.S. Chivukula, H.-J. He, M. Kurachi, E.H. Simons and M. Tanabashi, Phys. Rev. D70 (2004) 075008;

T. Nagasawa and M. Sakamoto, Prog. Theor. Phys. 112 (2004) 629;

Ch. Schwinn, Phys. Rev. D69 (2004) 116005;

C. Csaki, C. Grojean, J. Hubisz, Y. Shirnan and J. Terning, Phys. Rev. D70 (2004) 015012. 


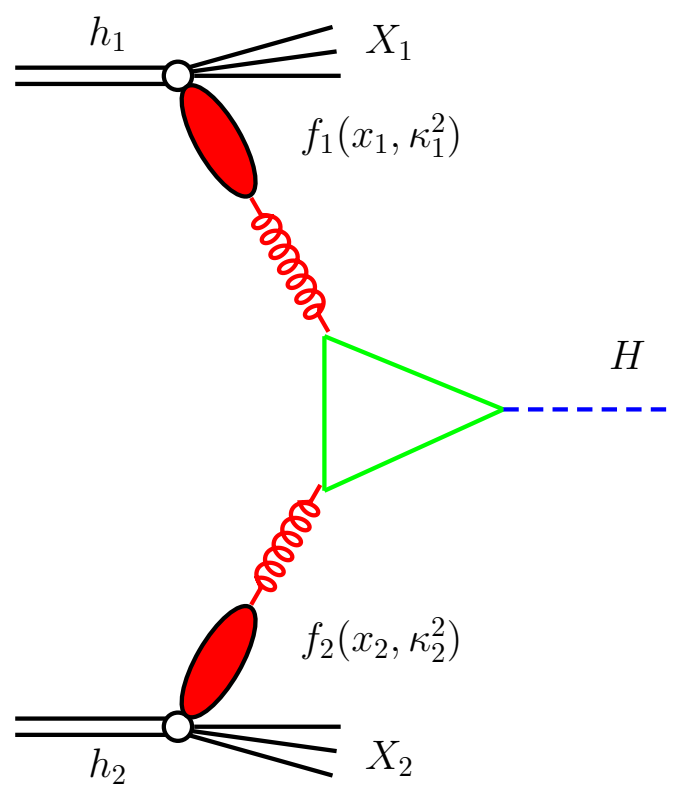

Figure 1: Dominant leading-order diagram for inclusive Higgs production for $p_{t} \ll M_{H}$. 


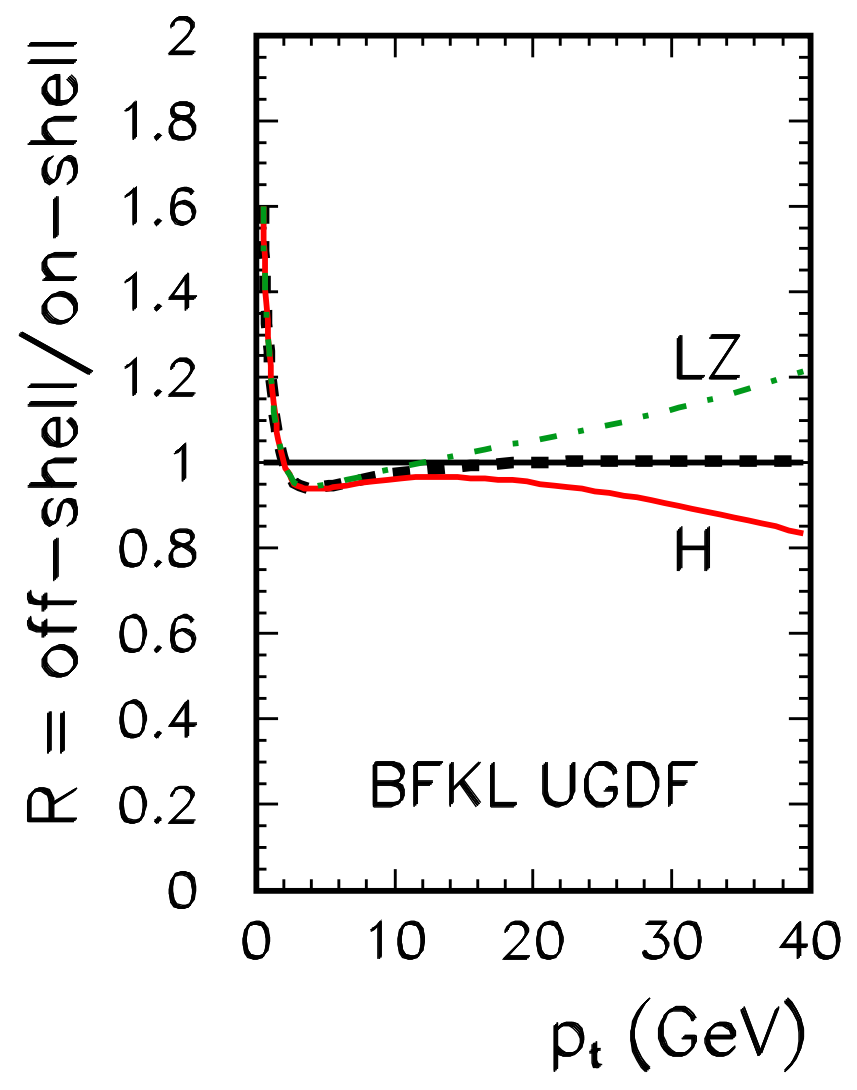

Figure 2: The ratio $R$ as a function of Higgs transverse momentum for the full range of Higgs rapidity. The thick dashed line corresponds to neglecting the function $\lambda$, i.e. assuming $\lambda=1$. The solid line was obtained with the Hautmann prescription of the flux factor [19] while the dash-dotted line is based on the formula from Ref.[20. In this calculation the BFKL UGDF was used as an example. 

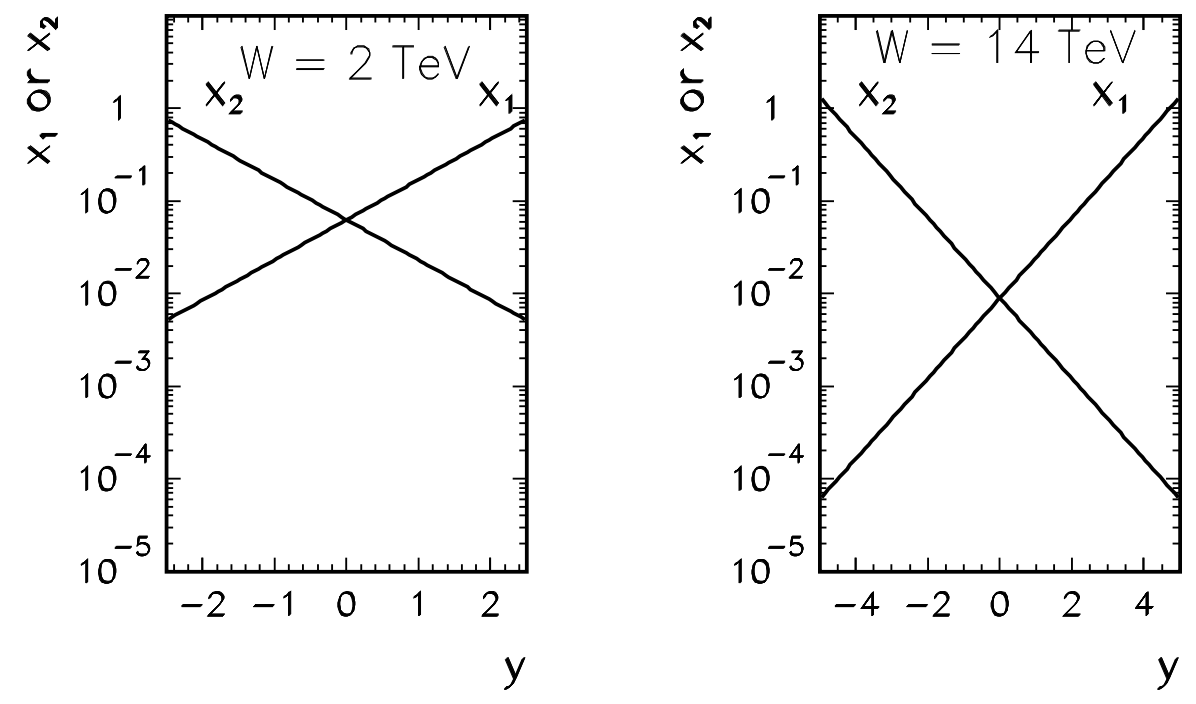

Figure 3: $x_{1}$ and $x_{2}$ as a function of Higgs rapidity $y$ for $p_{t}=0$. In panel (a) for Tevatron energy $\sqrt{s}=2$ TeV and in panel (b) for LHC energy $\sqrt{s}=$ $14 \mathrm{TeV}$. 


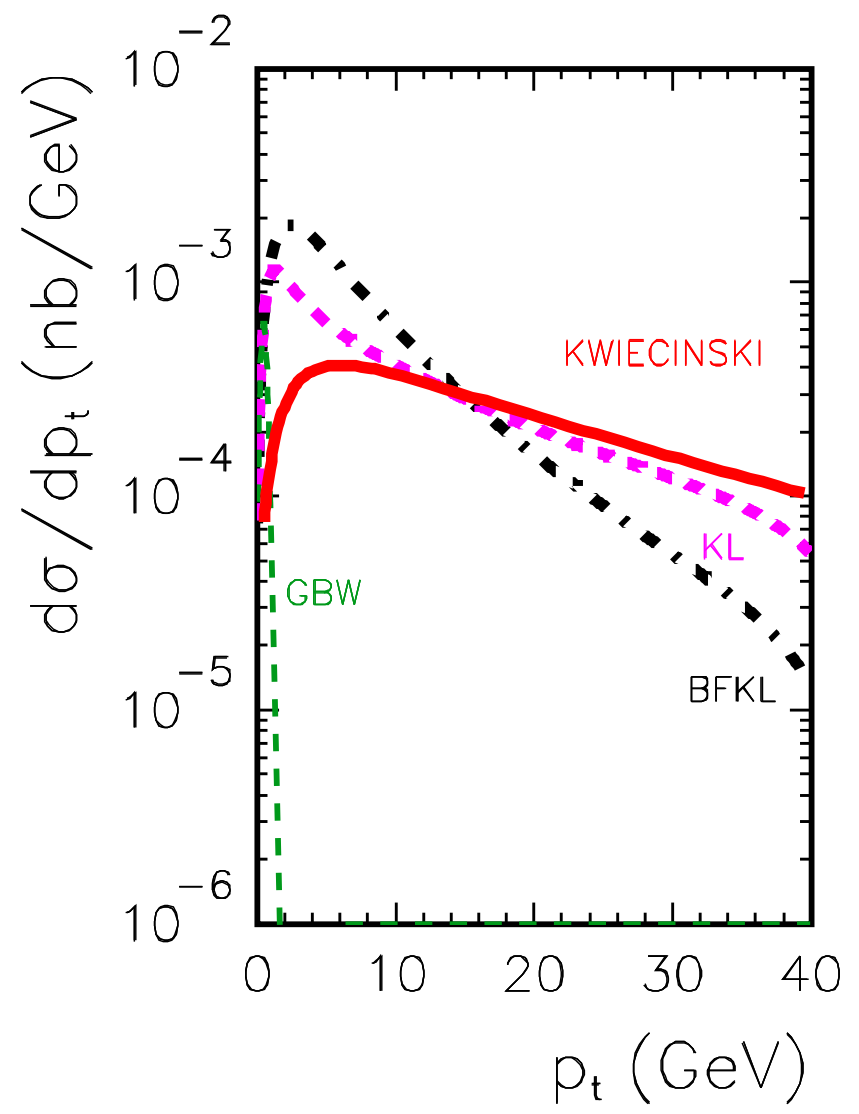

Figure 4: Transverse momentum distribution of Higgs boson at LHC energy $W=14 \mathrm{TeV}$ for different UGDF's from the literature: solid - Kwieciński, thick dashed -KL, thin dashed-GBW, dash-dotted-BFKL. 

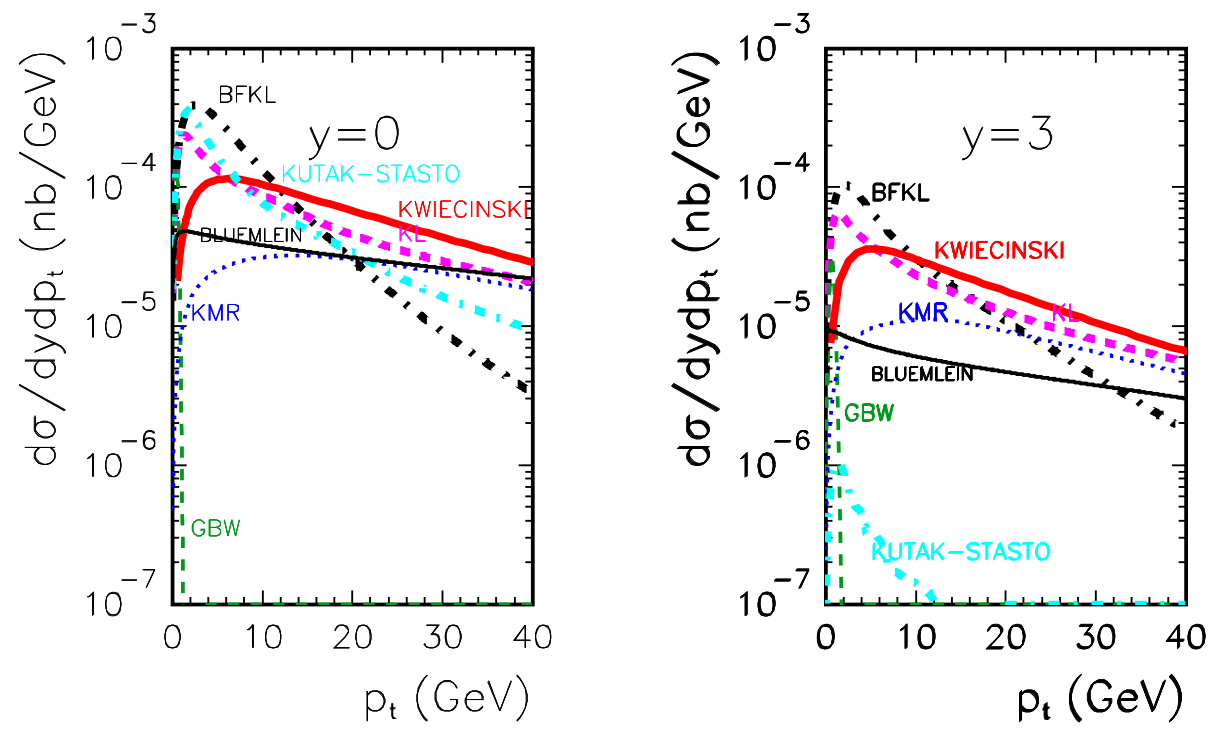

Figure 5: Transverse momentum distribution of Higgs boson at LHC energy $W=14 \mathrm{Te} V$ and $y=0$ (left panel) and $y= \pm 3$ (right panel) for different $U G D F$ 's from the literature. The notation here is the same as in the previous figure. In addition to the previous figure we present results for KMR (dotted), Kutak-Staśto (grey dash-dotted) and Bluemlein (thin solid) UGDF. 

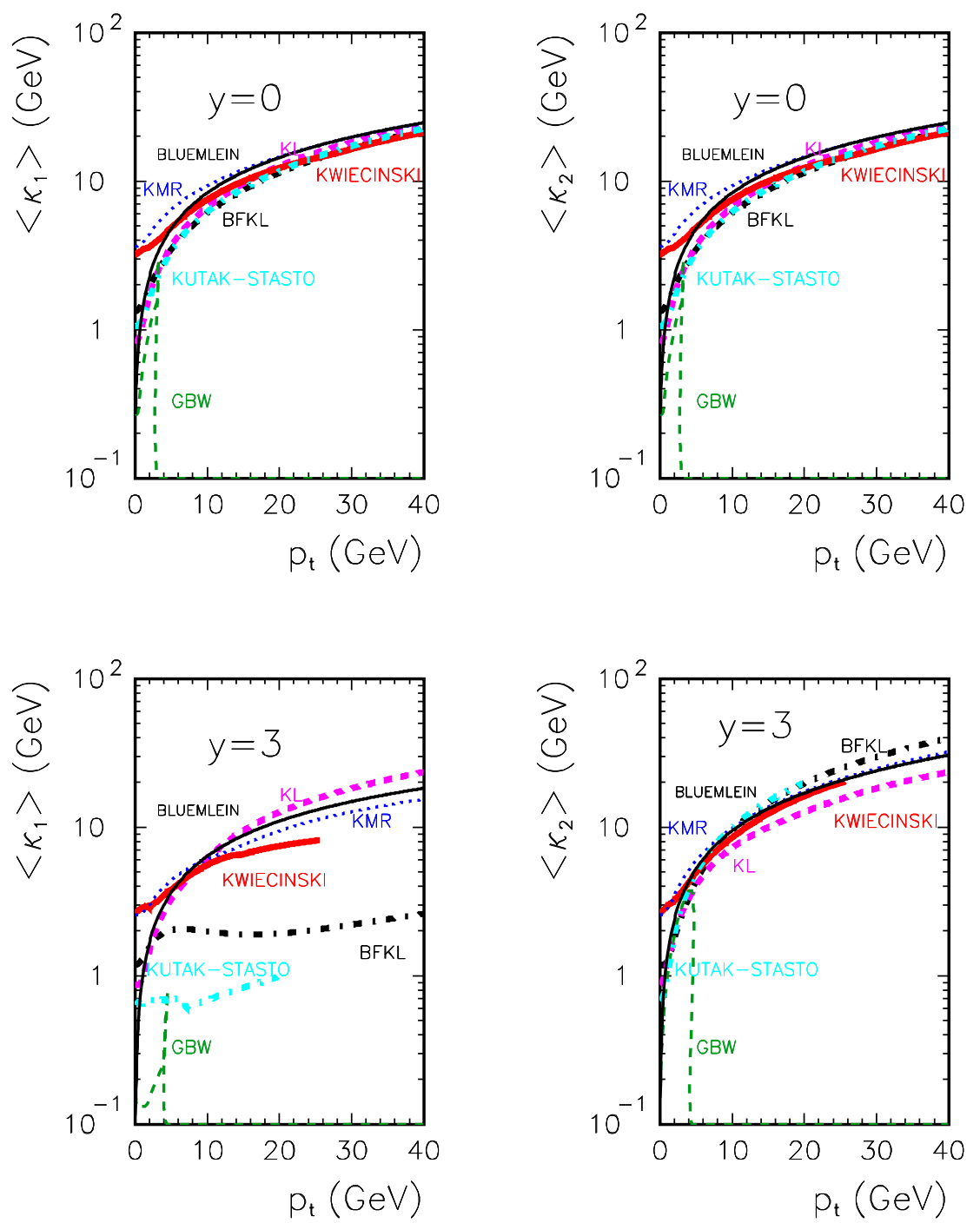

Figure 6: Average gluon transverse momentum as a function of Higgs transverse momentum at LHC energy $W=14 \mathrm{TeV}$ for different UGDF's from the literature. 


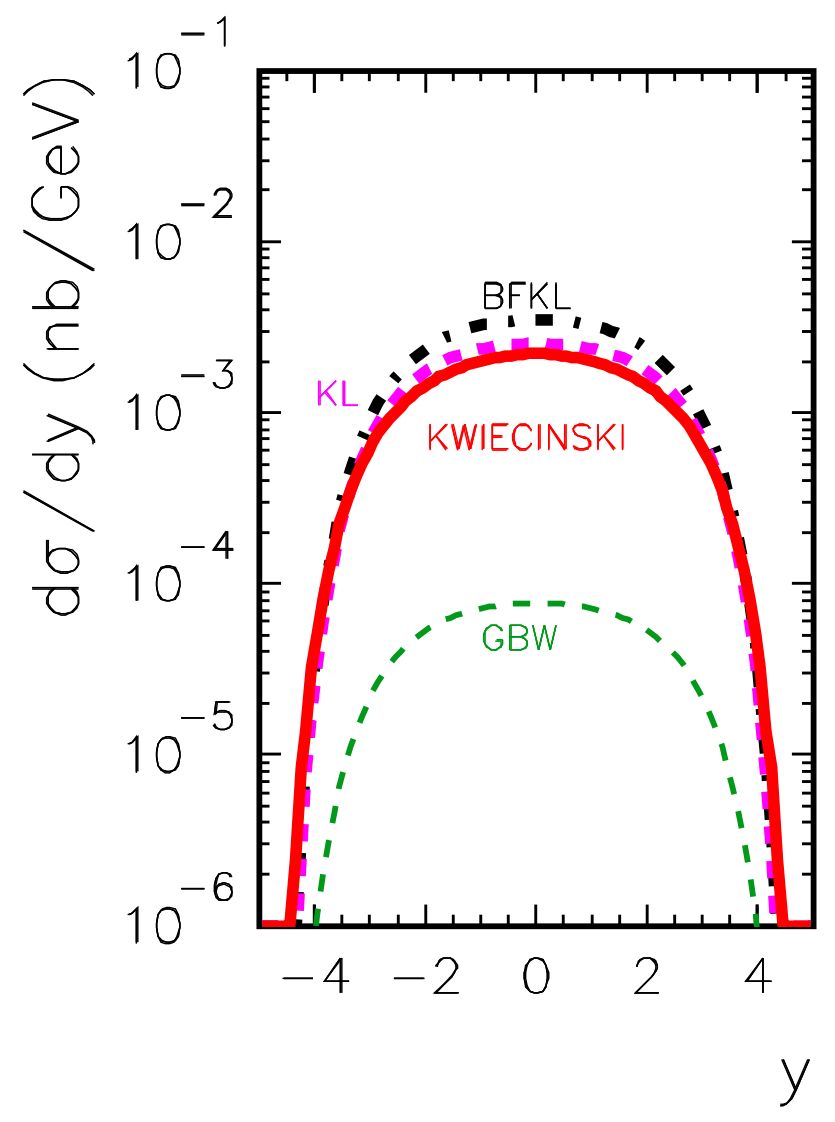

Figure 7: Rapidity distribution of Higgs at LHC energy $W=14 \mathrm{TeV}$ for different UGDF's from the literature. 

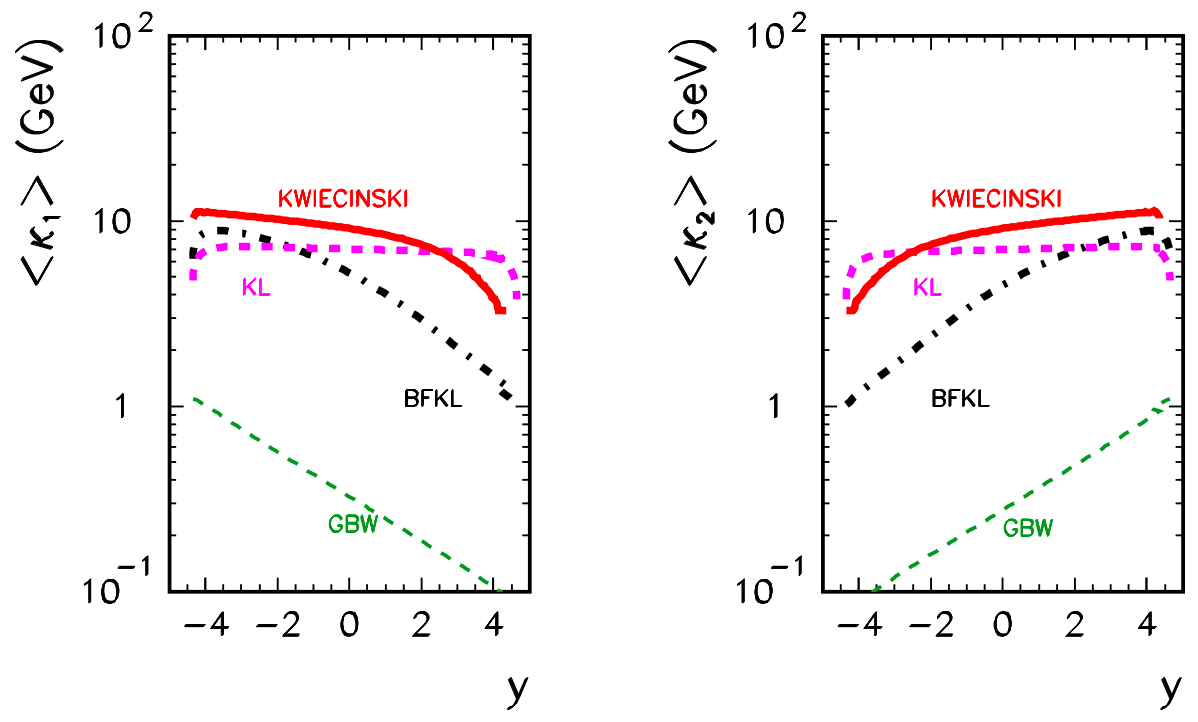

Figure 8: Average gluon transverse momentum $<\kappa_{1}>$ or $\left\langle\kappa_{2}>\right.$ as a function of Higgs rapidity for different UGDF's from the literature. In this calculation $p_{t}<40 \mathrm{GeV}$. 

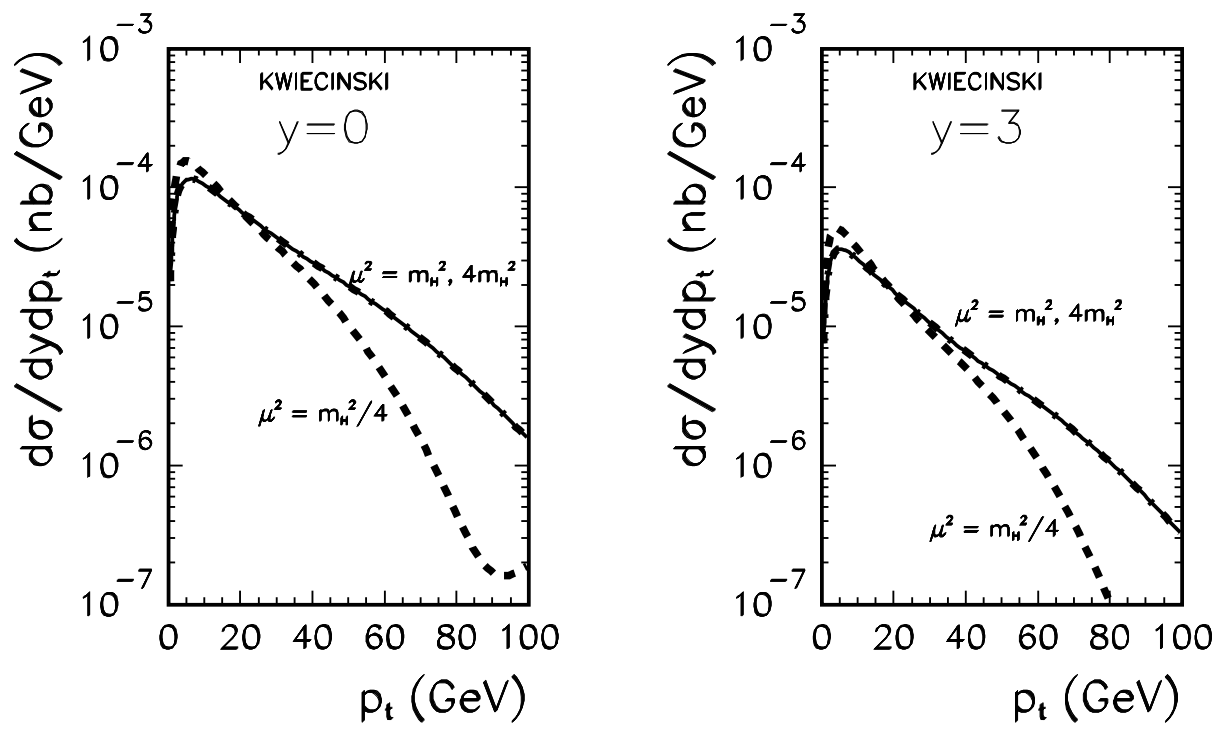

Figure 9: A dependence of the result on the choice of the factorization scale for the Kwiecinski UGDF for $y=0$ (left panel) and $y=3$ (right panel). In this calculation a Gaussian form factor with $b_{0}=1 G e V^{-1}$ was used. 

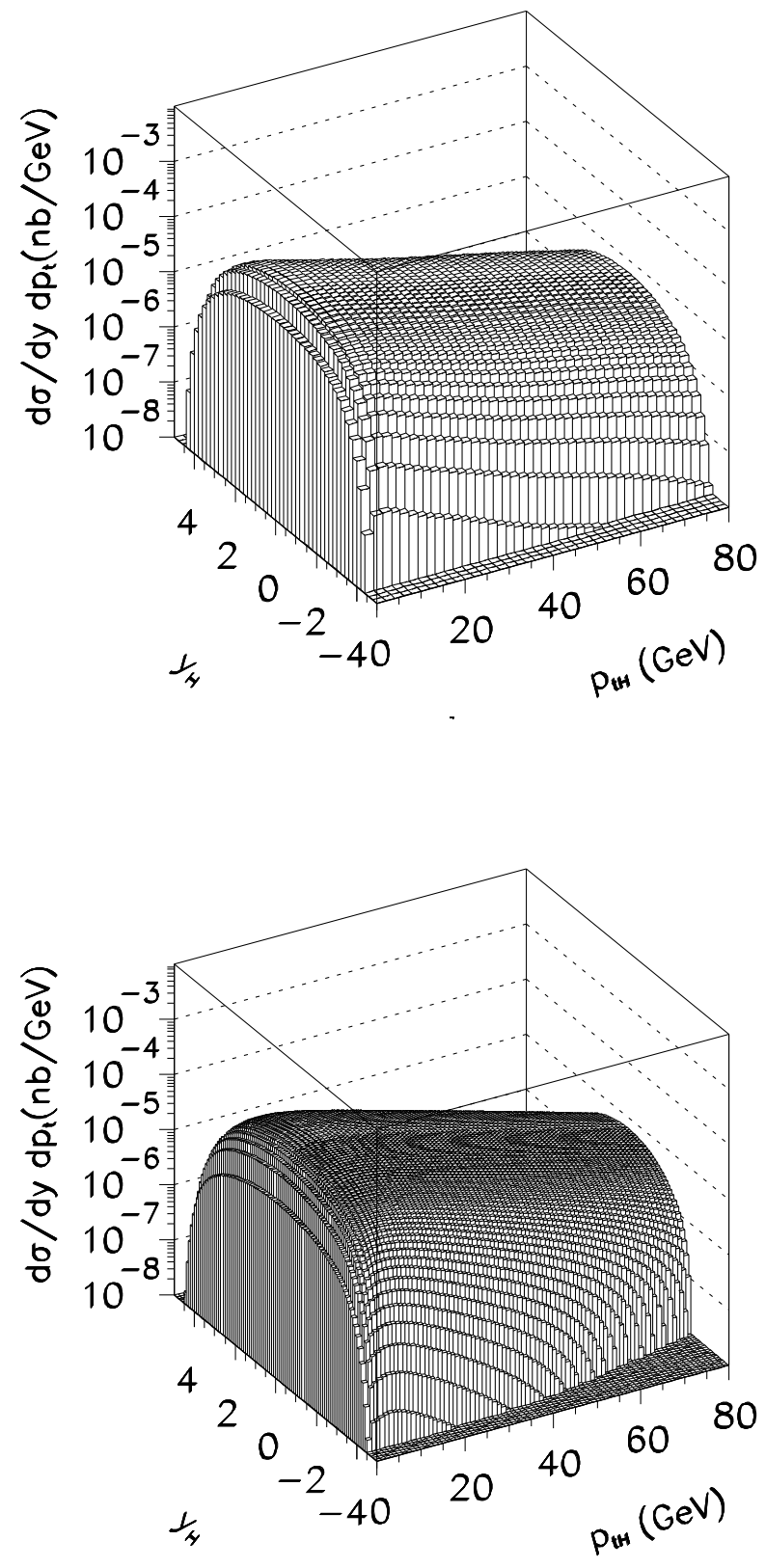

Figure 10: A comparison of two-dimensional distributions of Higgs boson for (a) Kwieciński UGDF, (b) LO b-space resummation. 

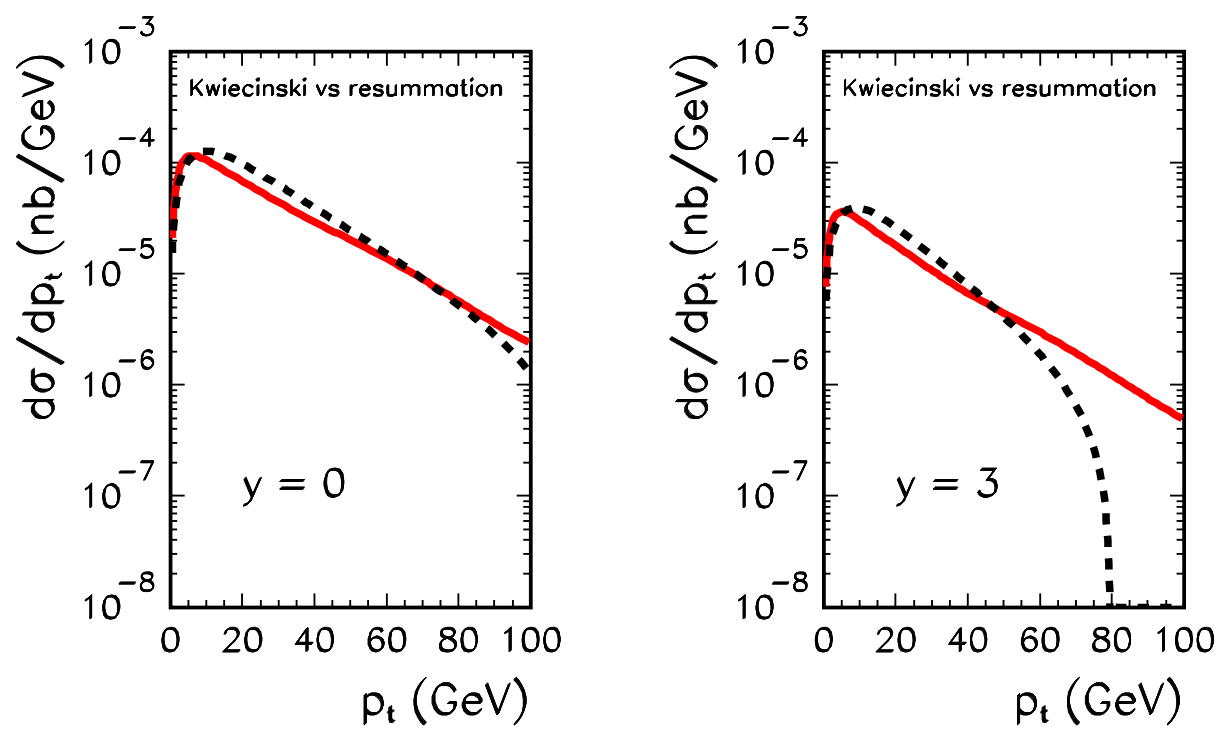

Figure 11: A comparison of Higgs transverse momentum distribution calculated with Kwiecinski UGDF (red, solid) and LO b-space resummation (black, dashed) for different rapidities: $y=0$ (left panel) and $y=3$ (right panel). 

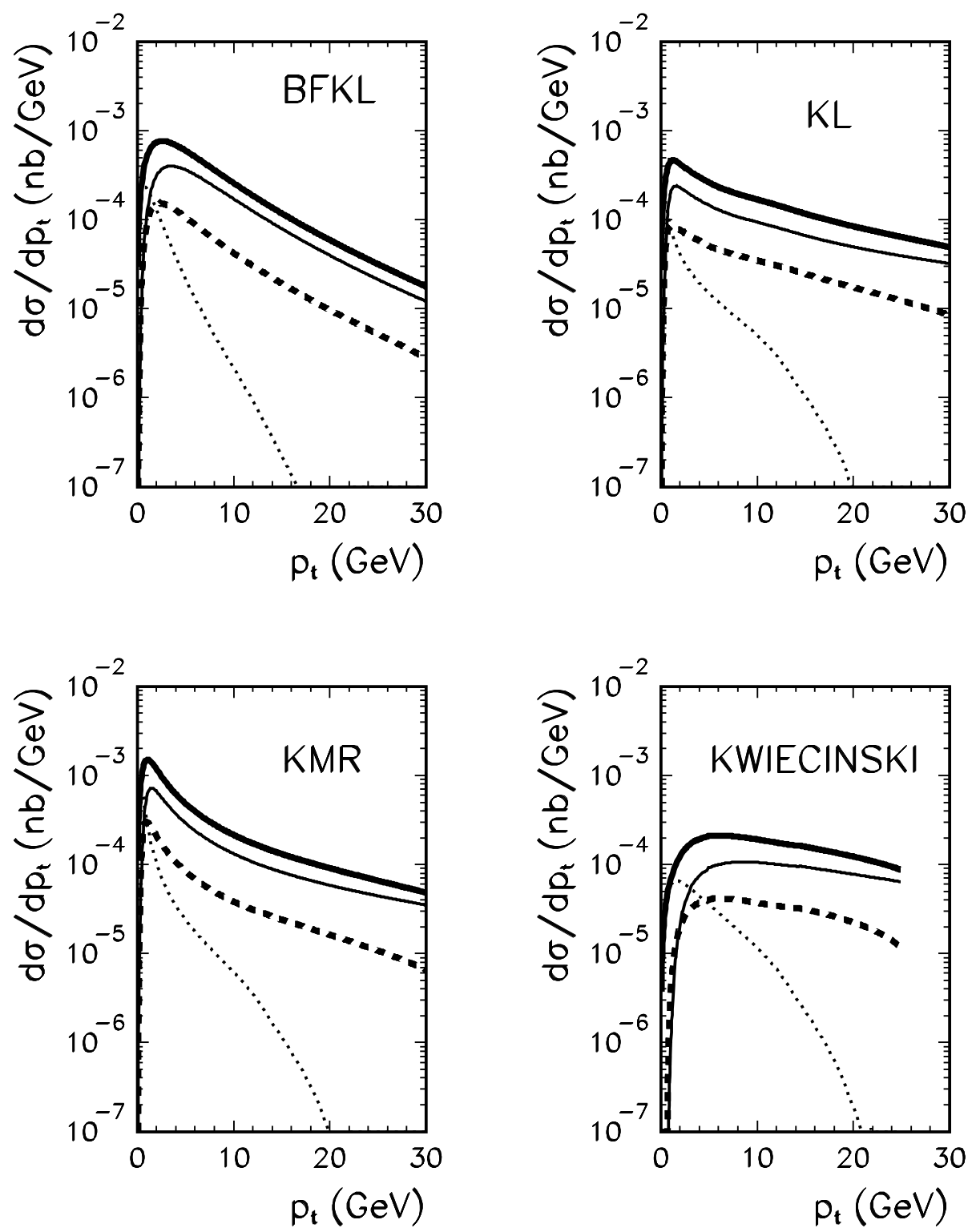

Figure 12: Decomposition of the transverse momentum distribution of Higgs boson at LHC energy $W=14 \mathrm{TeV}$ and $-1<y<1$ into four regions specified in the text. The thick solid line is a sum of all 4 contributions, thin solidregion I, dashed - region II+III) and dotted - region IV. 

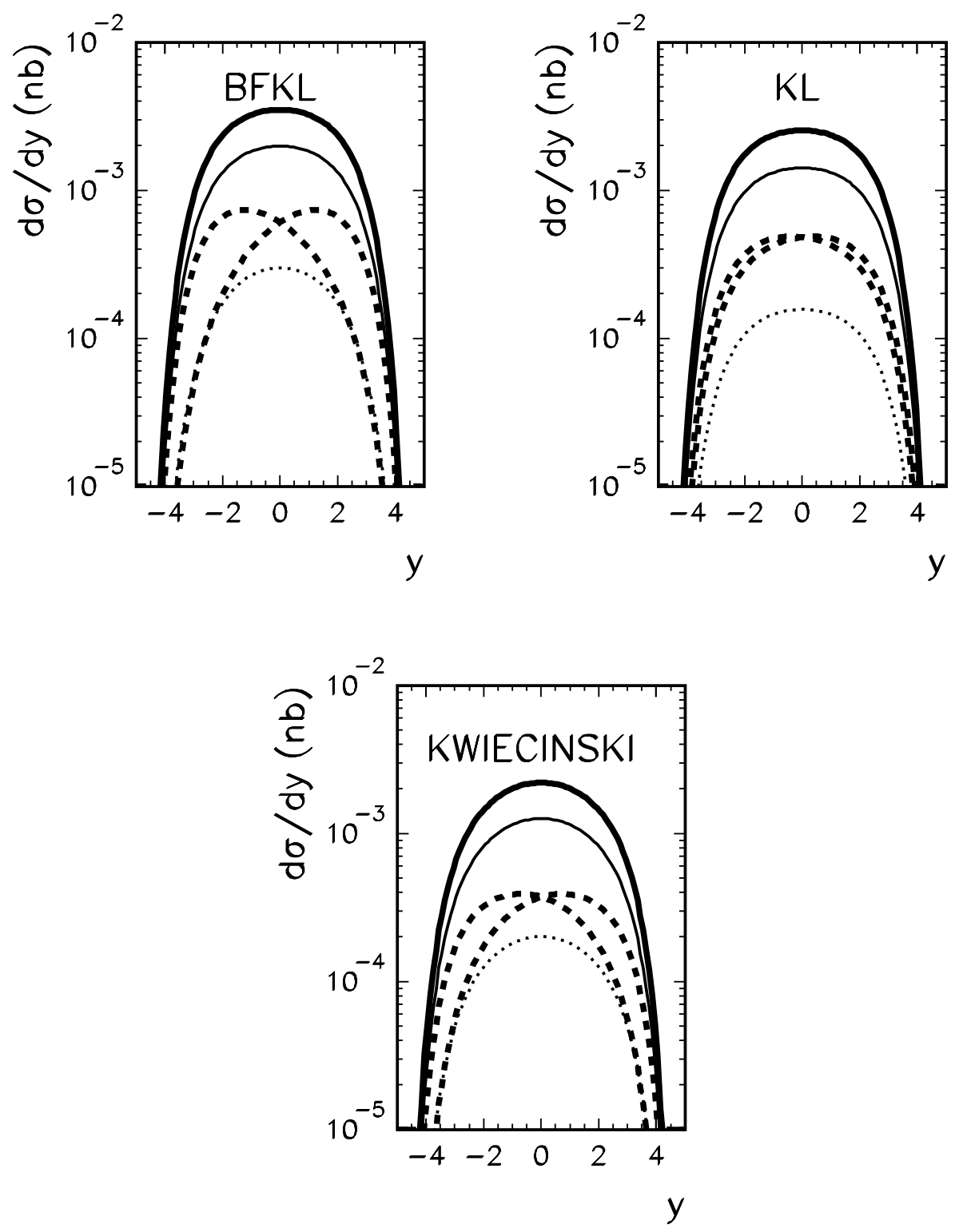

Figure 13: Decomposition of the rapidity distribution of Higgs boson at LHC energy $W=14 \mathrm{TeV}$ and into four regions specified in the text. In this calculation $p_{t}<40 \mathrm{GeV}$. The thick solid line is a sum of all 4 contributions, thin solid - region I, dashed - region II or III) and dotted - region IV. 

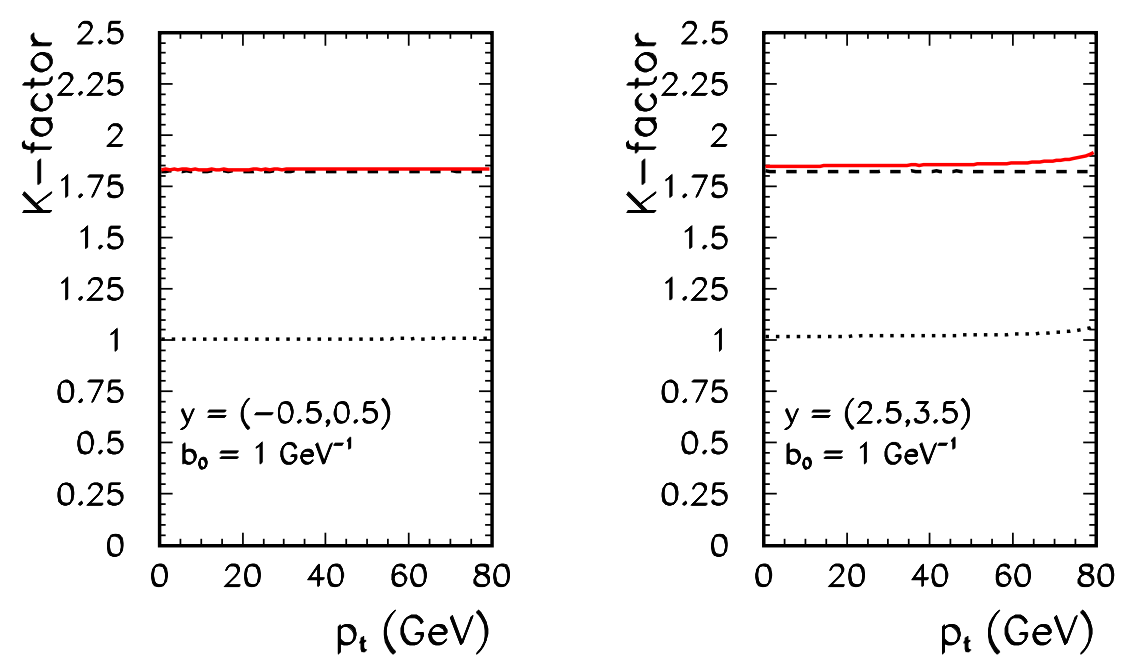

Figure 14: NLO K-factor in the soft-gluon-resummation formalism as a function of Higgs transverse momentum for two different bins of rapidity. The dashed line corresponds to separated gluonic contributions, while the dotted line to separated quarkish contributions. The solid line is for both effects included simultaneously. 


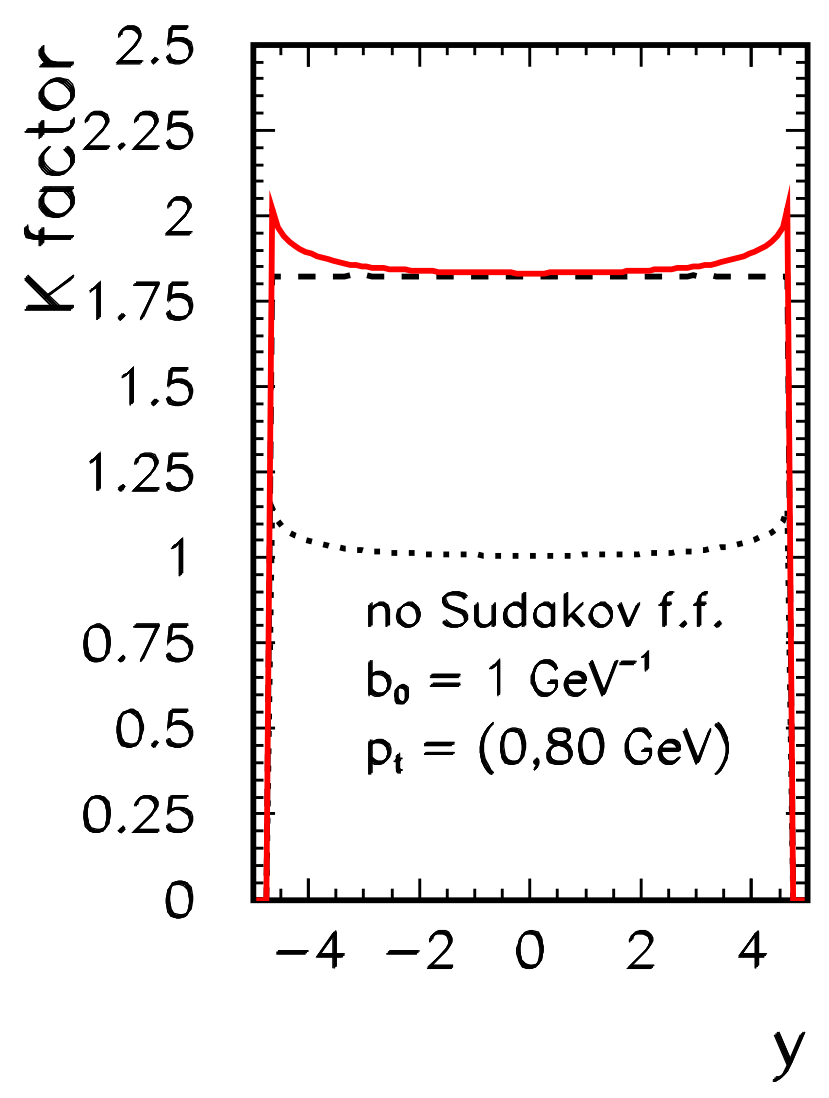

Figure 15: NLO K-factor in the soft-gluon-resummation formalism as a function of Higgs rapidity. In this calculation $p_{t}<80 \mathrm{GeV}$. The meaning of the curves here is the same as in the previous figure. 

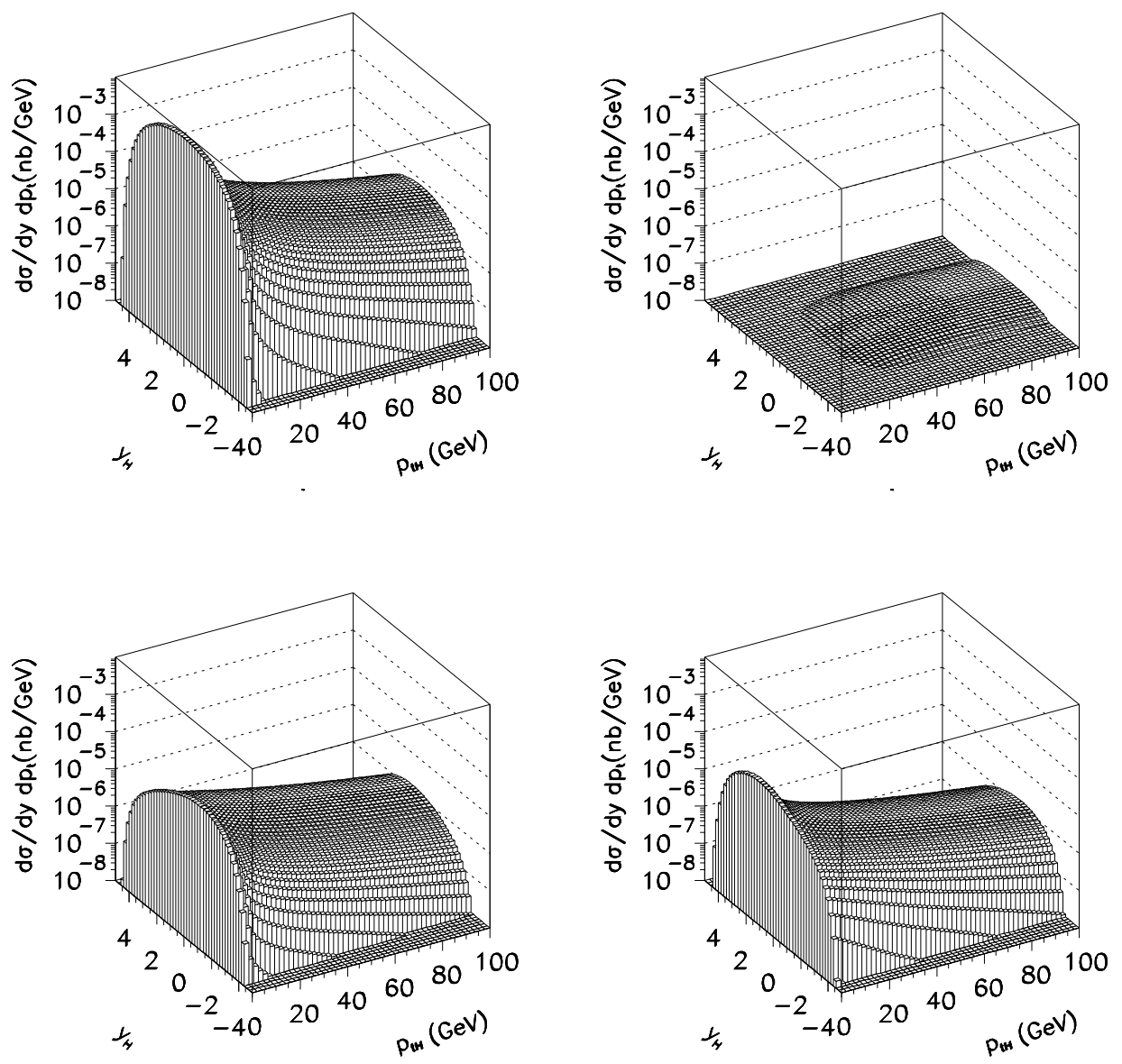

Figure 16: Contributions of different subprocesses of the $2 \rightarrow 2$ type for $W$ $=14 \mathrm{TeV}$, respectively for (a) $g g$, (b) $q q$, (c) $q g$ and (d) $g q$. 


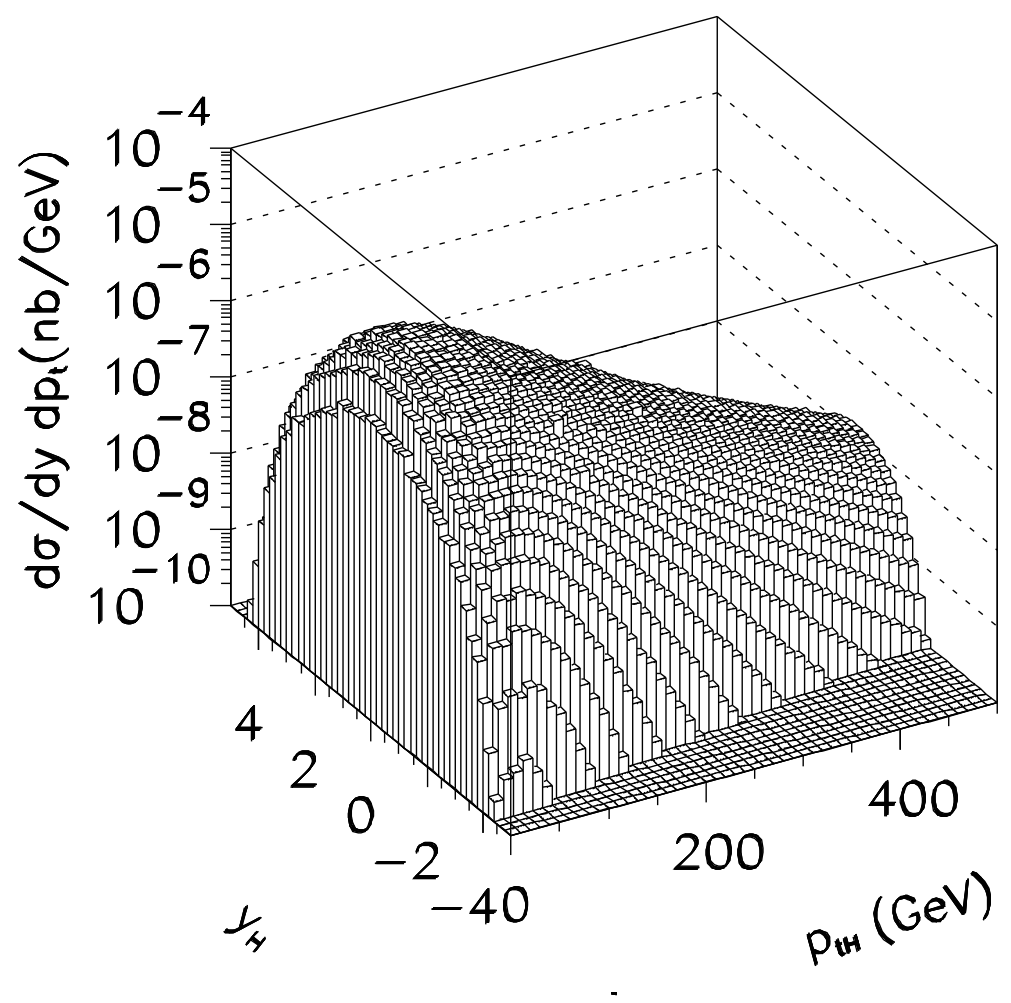

Figure 17: Two-dimensional distribution in $y$ and $p_{t}$ of Higgs from $L O W W$ fusion process. In this calculation we have taken $\mu_{1}^{2}=\mu_{2}^{2}=M_{H}^{2}$. 

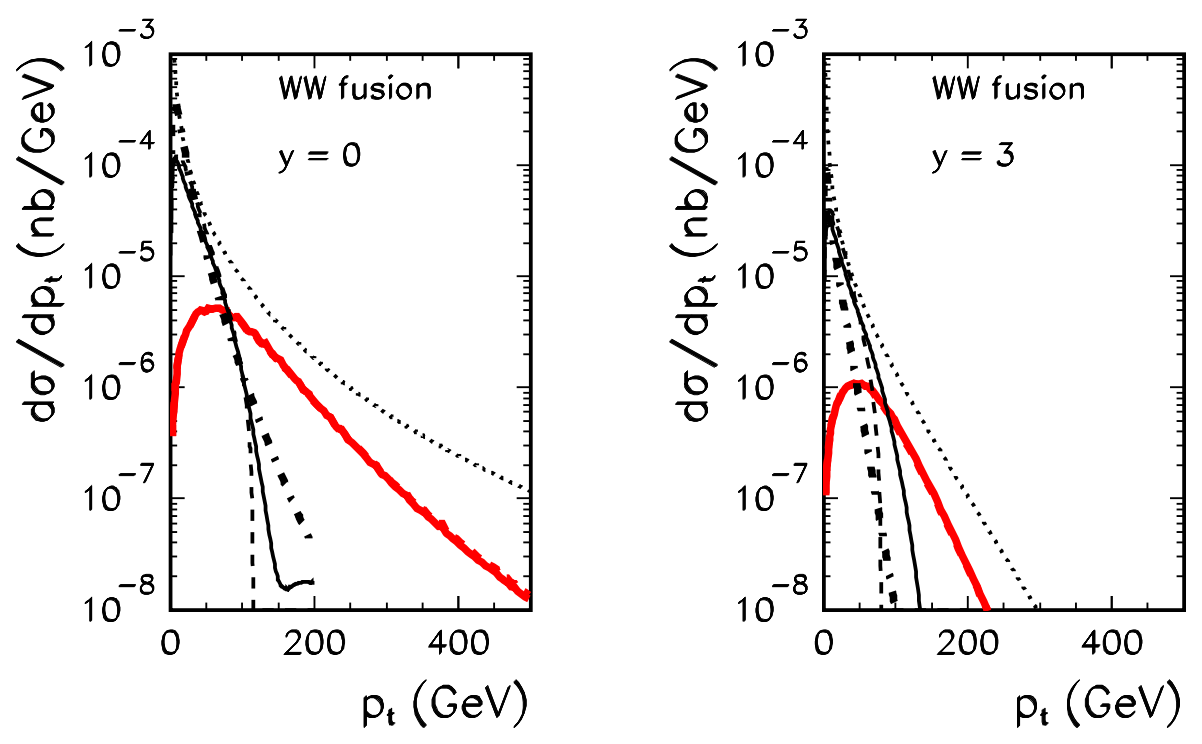

Figure 18: Transverse momentum distribution of Higgs boson at LHC energy $W=14$ TeV and $y=0$ (left panel) and $y= \pm 3$ (right panel) produced in $W W$ fusion (overlaping thick solid and thick dashed for the two different prescriptions specified in the formalism section), compared to the corresponding contribution of gluon-gluon fusion: BFKL (dash-dotted), Kwiecinski (thin solid) UGDF, LO soft-gluon resummation (dashed) and the perturbative $2 \rightarrow$ 2 collinear contribution (thin dotted). 


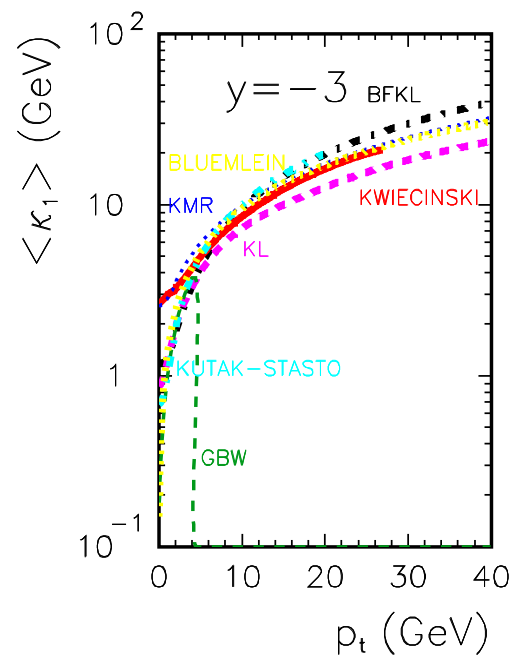




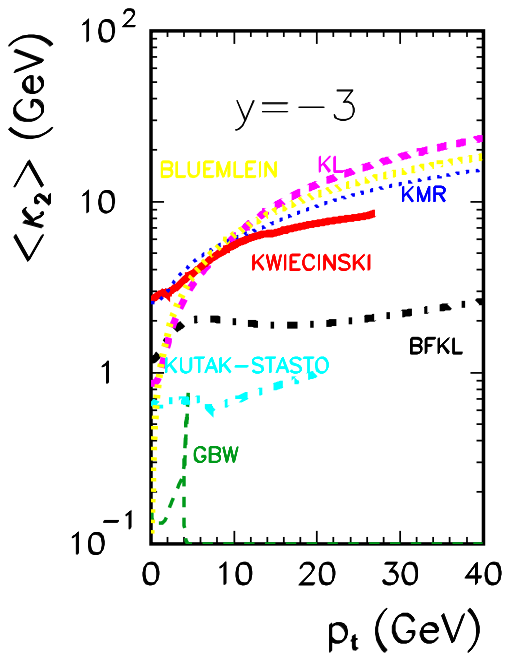

